\title{
Invariants of welded virtual knots via crossed module invariants of knotted surfaces
}

\author{
Louis H. Kauffman and João Faria Martins
}

\begin{abstract}
We define an invariant of welded virtual knots from each finite crossed module by considering crossed module invariants of ribbon knotted surfaces which are naturally associated with them. We elucidate that the invariants obtained are non-trivial by calculating explicit examples. We define welded virtual graphs and consider invariants of them defined in a similar way.
\end{abstract}

\section{Introduction}

Welded virtual knots were defined in [Kau99], extending the analogous construction of welded braids in [FRR97], by allowing one extra move in addition to the moves appearing in the definition of a virtual knot. This extra move preserves the (combinatorial) fundamental group of the complement, which is therefore an invariant of welded virtual knots (the knot group). Given a finite group $G$, one can therefore define a welded virtual knot invariant $\mathcal{H}_{G}$, by considering the number of morphisms from the fundamental group of the complement into $G$. The Wirtinger presentation of knot groups enables a quandle-type calculation of this 'counting invariant' $\mathcal{H}_{G}$.

Not a lot of welded virtual knot invariants are known. The aim of this paper is to introduce a new one, the 'crossed module invariant' $\mathcal{H}_{\mathcal{G}}$, which depends on a finite automorphic crossed module $\mathcal{G}=(E, G, \triangleright)$, in other words on a pair of groups $E$ and $G$, with $E$ abelian, and a left action of $G$ on $E$ by automorphisms.

The crossed module invariant $\mathcal{H}_{\mathcal{G}}$ reduces to the counting invariant $\mathcal{H}_{G}$ when $E=0$. However, the crossed module invariant distinguishes, in some cases, between welded virtual links with the same knot group, and therefore it is strictly stronger than the counting invariant. We will assert this fact by calculating explicit examples.

Let $\mathcal{G}=(E, G, \triangleright)$ be an automorphic crossed module. Note that the counting invariant $\mathcal{H}_{G}$ is trivial whenever $G$ is abelian. However, taking $G$ to be abelian and $E$ to be non-trivial yields a non-trivial invariant $\mathcal{H}_{\mathcal{G}}$, which is, as a rule, much easier to calculate than the counting invariant $\mathcal{H}_{G}$ where $G$ is a generic group, and it is strong enough to tell apart some pairs of links with the same knot group. Suppose that the welded virtual link $K$ has $n$ components. Let $\kappa_{n}=\mathbb{Z}\left[X_{1}, X_{1}^{-1}, \ldots, X_{n}, X_{n}^{-1}\right]$. We will define a $k_{n}$-module $\mathrm{CM}(K)$, depending only on $K$, up to isomorphism and permutations of the variables $X_{1}, \ldots, X_{n}$. If $G$ is abelian, then $\mathcal{H}_{\mathcal{G}}$ simply counts the number of crossed module morphisms $\operatorname{CM}(K) \rightarrow \mathcal{G}$. We prove in this paper that if $K$ is classical then $\operatorname{CM}(K)$ coincides with the Alexander module Alex $(K)$ of $K$. However, this is not the case if $K$ is not classical. We will give examples of pairs of welded virtual links $\left(K, K^{\prime}\right)$ with the same knot group (thus the same Alexander module) but with $\operatorname{CM}(K) \nRightarrow \operatorname{CM}\left(K^{\prime}\right)$. This will happen when $K$ and $K^{\prime}$ have the same knot group, but are distinguished by their crossed module invariants for $G$ abelian.

Received 30 April 2007, accepted in final form 9 December 2007.

2000 Mathematics Subject Classification 57M25 (primary), 57Q45 (secondary).

Keywords: welded virtual knots, knotted surfaces, crossed module, quandle invariants, Alexander module.

This journal is (C) Foundation Compositio Mathematica 2008. 


\section{INVARIANTS OF WELDED VIRTUAL KNOTS}

Let us explain the construction of the crossed module invariant $\mathcal{H}_{\mathcal{G}}$. Extending a previous construction due to Yagima, Satoh defined in [Sat00] a map which associates an oriented knotted torus $T(K)$, the 'tube of $K$ ', to each oriented welded virtual knot $K$. The map $K \mapsto T(K)$ preserves knot groups. In the case when $K$ is a classical knot, then $T(K)$ coincides with the torus spun of $K$, obtained by spinning $K$ four-dimensionally, in order to obtain an embedding of the torus $S^{1} \times S^{1}$ into $S^{4}$.

The existence of the tube map $K \mapsto T(K)$ makes it natural to define invariants of welded virtual knots by considering invariants of knotted surfaces. We will consider this construction for the case of the crossed module invariants $I_{\mathcal{G}}(\Sigma)$ of knotted surfaces $\Sigma$, defined in [FM07a, FM08]. Here $\mathcal{G}=(E \stackrel{\stackrel{\partial}{\rightarrow}}{\rightarrow}, \triangleright)$ is a finite crossed module. Note that the invariant $I_{\mathcal{G}}$ on a knotted surface coincides with Yetter's invariant (see [Yet93, Por96, FMP07]) of the complement of it. We can thus define a welded virtual knot invariant by considering $\mathcal{H}_{\mathcal{G}}(K) \doteq I_{\mathcal{G}}(T(K))$, where $K$ is a welded virtual knot.

A straightforward analysis of the crossed module invariant of the tube $T(K)$ of the welded virtual knot $K$ permits the evaluation of $\mathcal{H}_{\mathcal{G}}(K)$ in a quandle-type way, albeit the biquandle we define is sensitive to maximal and minimal points, so it should probably be called a 'Morse biquandle'.

A proof of the existence of the invariant $\mathcal{H}_{\mathcal{G}}$, where $\mathcal{G}$ is a finite crossed module, can be done directly, from the Morse biquandle obtained. In fact all the results of this paper are fully independent of the four-dimensional picture, and can be given a direct proof. Moreover, they confirm the results obtained previously for the crossed module invariants $I_{\mathcal{G}}$ of knotted surfaces in $S^{4}$.

As we have referred to above, the tube map $K \mapsto T(K)$ preserves the fundamental group of the complements. We prove that $\mathcal{H}_{\mathcal{G}}$ is powerful enough to distinguish between distinct welded virtual links with the same knot group. For example, we will construct an infinite set of pairs $\left(P_{i}, c_{1}\left(P_{i}^{\prime}\right)\right)$, where $i$ is an odd integer, of welded virtual links with the following properties:

(i) $P_{i}$ and $c_{1}\left(P_{i}^{\prime}\right)$ each have two components for all $i$;

(ii) $P_{i}$ and $c_{1}\left(P_{i}^{\prime}\right)$ have isomorphic knot groups for each $i$;

(iii) $P_{i}$ and $c_{1}\left(P_{i}^{\prime}\right)$ can be distinguished by their crossed module invariant for each $i$.

In fact $P_{i}$ and $c_{1}\left(P_{i}^{\prime}\right)$ will be distinguished by their crossed module invariant $\mathcal{H}_{\mathcal{G}}$ with $\mathcal{G}=(E, G, \triangleright)$ being an automorphic crossed module with $G$ abelian. This in particular proves that the crossed module invariant of knotted surfaces $I_{\mathcal{G}}$ defined in [FM07a, FM08, FM07b] sees beyond the fundamental group of their complement, in an infinite number of cases.

We will also give examples of pairs of one-component welded virtual knots with the same knot group, but separated by their crossed module invariants. However, we will need to make use of computer-based calculations in this case.

In this paper we will propose a definition of welded virtual graphs. The crossed module invariant of welded virtual links extends naturally to them.

\section{An invariant of welded virtual knots}

\subsection{Welded virtual knots}

Recall that a virtual knot diagram is, by definition, an immersion of a disjoint union of circles into the plane $\mathbb{R}^{2}$, where the 4 -valent vertices of the immersion can represent either classical or virtual crossing; see Figure 1. The definition of an oriented virtual knot diagram is the obvious one. We say that two virtual knot diagrams are equivalent if they can be related by the moves of Figures 2 and 3, as well as planar isotopy. It is important to note that in the oriented case we will need to consider all the possible orientations of the strands. A virtual knot is an equivalence class of virtual knot diagrams under the equivalence relation just described; see [Kau99]. 
L. H. Kauffman And J. FARia Martins
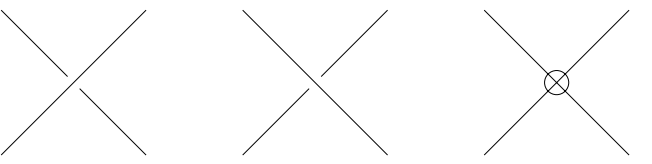

Figure 1. Classical and virtual crossings.
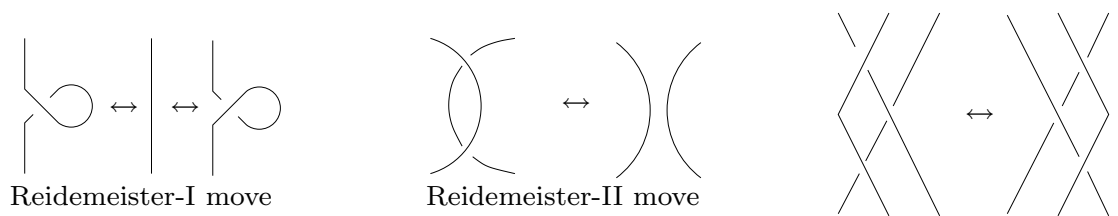

Reidemeister-III move

FiguRE 2. Reidemeister moves I, II and III.
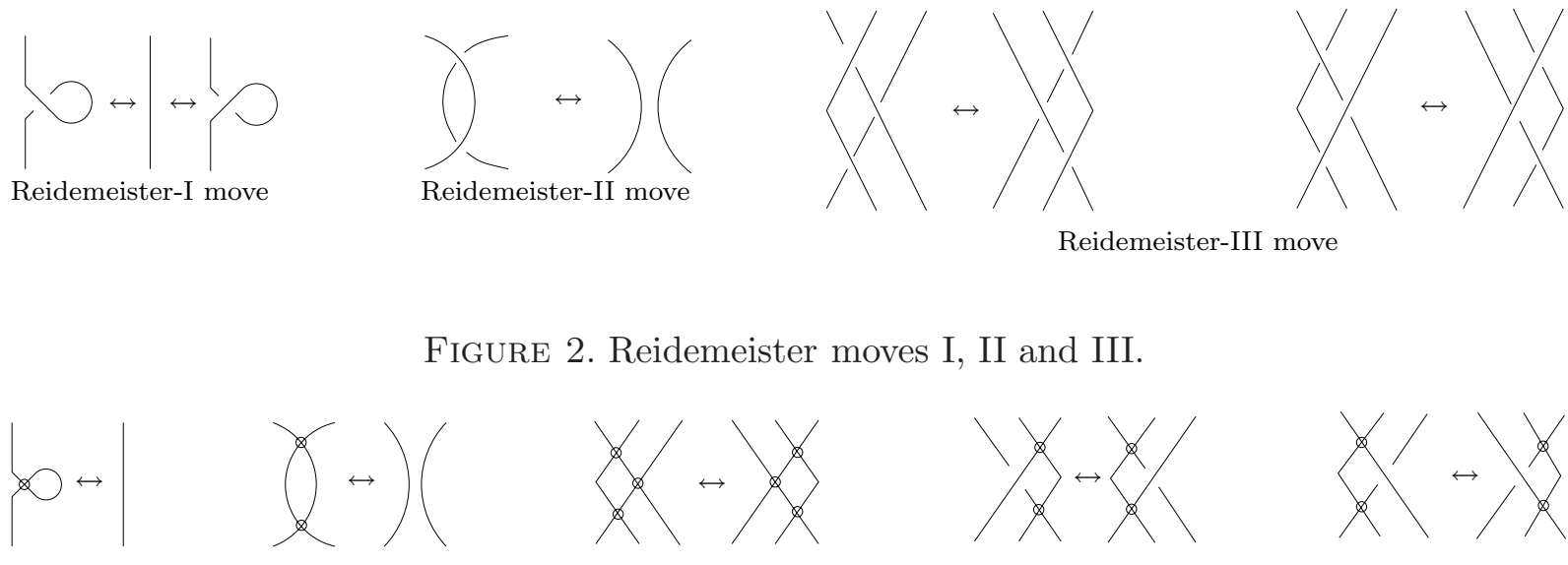

Figure 3. Virtual Reidemeister moves.
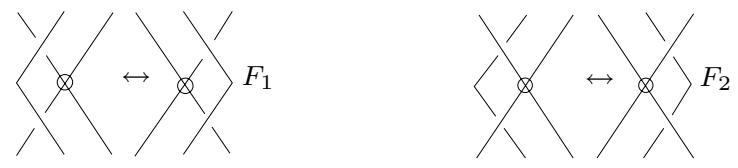

FiguRE 4 . The forbidden moves $F_{1}$ and $F_{2}$.
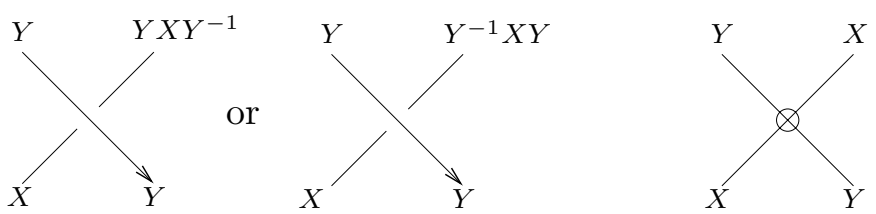

FiguRE 5. Wirtinger relations. The first two are called 'left handed' and 'right handed' Wirtinger relations, respectively.

Observe that, as far as virtual knots are concerned, we do not allow the moves shown in Figure 4, called respectively the forbidden moves $F_{1}$ and $F_{2}$. Considering the first forbidden move $F_{1}$ in addition to the ones appearing in the definition of a virtual knot, one obtains the notion of a 'welded virtual knot', due to the first author; see [Kau99].

2.1.1 The fundamental group of the complement. The (combinatorial) fundamental group of the complement of a virtual knot diagram (the knot group) is, by definition, generated by all the arcs of a diagram of it, considering the relations (called Wirtinger relations) of Figure 5 at each crossing. It is understood that in each calculation of a knot group from a virtual knot diagram we will use either the 'left handed' or the 'right handed' Wirtinger relation. The final result will not depend on this choice. 


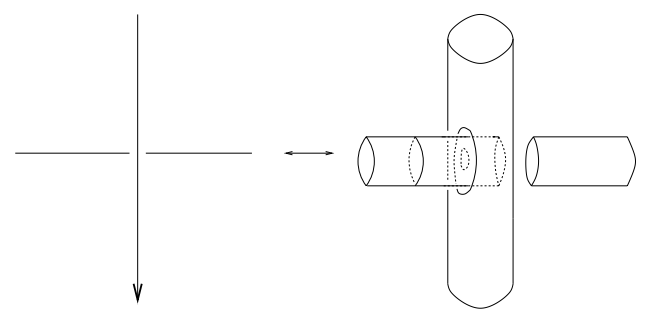

Figure 6 . The tube of a virtual knot in the vicinity of a classical crossing.
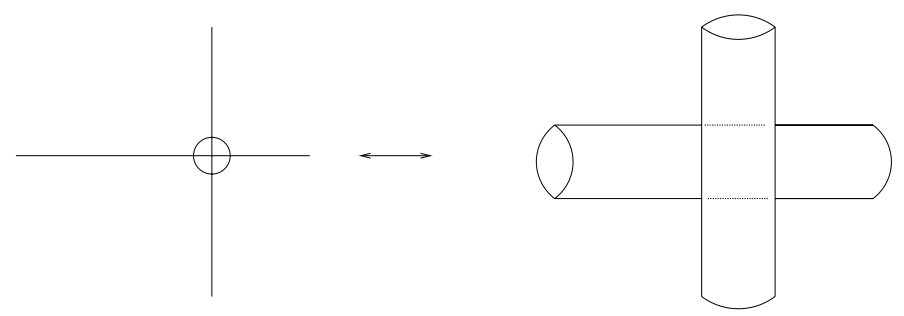

Figure 7 . The tube of a virtual knot in the vicinity of a virtual crossing.

In the case of classical knots or links, this does coincide with the fundamental group of the complement, so we can drop the prefix 'combinatorial'. This combinatorial fundamental group is in fact an invariant of welded virtual knots. This can be proved easily.

\subsection{Virtual knot presentations of knotted surfaces}

By definition, a torus $\operatorname{link}^{1}$ in $S^{4}$ is an embedding of a disjoint union of tori $S^{1} \times S^{1}$ into $S^{4}$, considered up to ambient isotopy. A knotted torus is an embedding of a torus $S^{1} \times S^{1}$ into $S^{4}$, considered up to ambient isotopy. The definition of an oriented knotted torus or torus link is the obvious one.

As proved in [Sat00, Yaj62, CKS04], it is possible to associate an oriented torus link $T(K) \subset$ $S^{4}$, the 'tube of $K$ ', to each oriented welded virtual link $K$. This correspondence was defined first in [Yaj62], for the case of classical knots. The extension to welded virtual knots was completed in [Sat00].

The tube map is very easy to define. Given a virtual link diagram, we define the tube of it by considering the broken surface diagram obtained by doing the transition of Figures 6 and 7 . For the representation of knotted surfaces in $S^{4}$ in the form of broken surface diagrams, we refer the reader to [CKS04]. The tube of a virtual knot diagram has a natural orientation determined by the orientation of a ball in $S^{3}$. It is proved in [Sat00] that if $K$ and $L$ are diagrams of the same welded virtual knot then it follows that $T(K)$ and $T(L)$ are isotopic knotted surfaces in $S^{4}$. This defines the tube of a welded virtual knot.

For calculation purposes, however, it is important to have a definition of the 'tube map' in terms of movies. Let $D \subset \mathbb{R}^{2}$ be an oriented virtual knot diagram. We can suppose, apart from planar isotopy, that the projection on the second variable is a Morse function on $D$. Define a movie of a knotted surface by using the correspondence of Figures 8, 9 and 10. Note our convention of reading movies of knotted surfaces from the bottom to the top. This yields an alternative way of describing the tube $T(K)$ of the virtual knot $K$, if we are provided with a diagram of it.

\footnotetext{
${ }^{1}$ Not to be confused with the three-dimensional notion of a torus link.
} 
L. H. Kauffman And J. FARia Martins

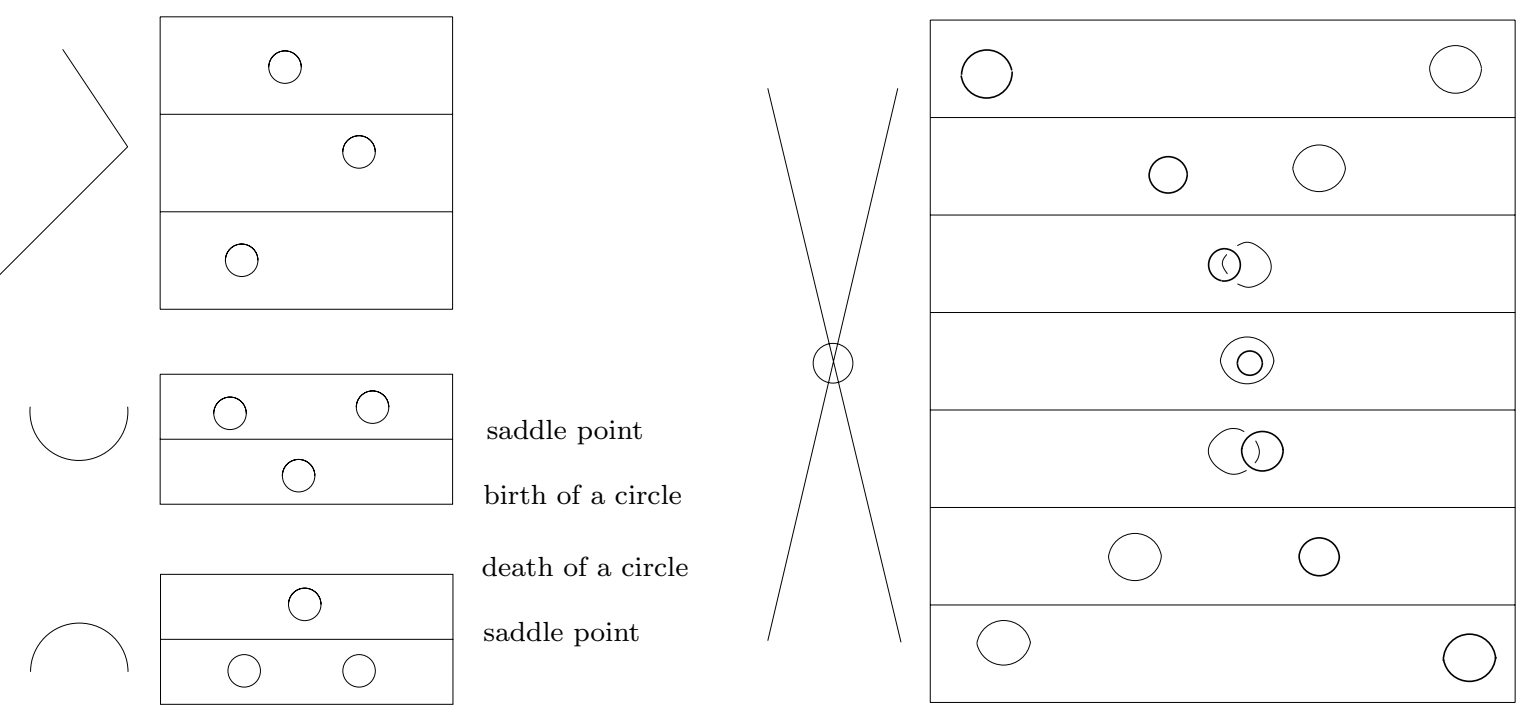

Figure 8. Associating a knotted torus to a virtual knot: edges, minimal and maximal points and virtual crossings. All circles are oriented counterclockwise. Note that the movies should be read from bottom to top.
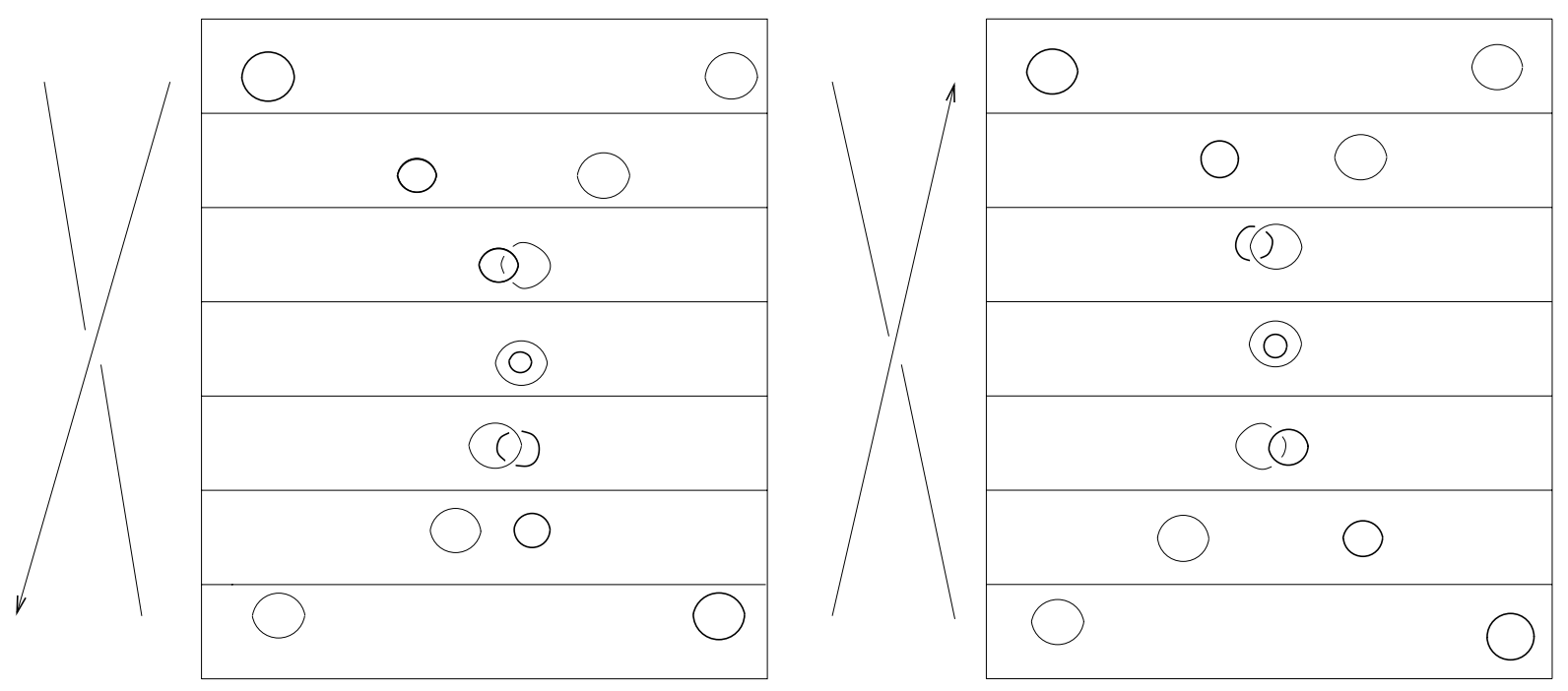

Figure 9. Associating a knotted torus to a virtual knot: classical crossing points, first case. All circles are oriented counterclockwise.

It was proved in [Sat00, Yaj62] that the correspondence $K \mapsto T(K)$, where $K$ is a welded virtual knot, preserves the fundamental groups of the complement (the knot groups).

Given a (classical) link $K$ with $n$ components sitting in the interior of the semiplane $\{(x, y, z) \in$ $\left.\mathbb{R}^{3}: z \geqslant 0\right\}$, we define the torus spun of $K$ by rotating $K$ four-dimensionally around the plane $\{z=0\}$. Therefore, we obtain an embedding of the disjoint union of $n$ tori $S^{1} \times S^{1}$ into $S^{4}$. It was shown in [Sat00] that the torus spun of $K$ is in fact isotopic to the tube $T(K)$ of $K$.

The correspondence $K \mapsto T(K)$ actually sends welded virtual links to ribbon torus links. In fact, any ribbon torus link is of the form $T(K)$ for some welded virtual knot $K$. However, it is an open problem whether the map $K \mapsto T(K)$ is faithful; see [CKS04, problems (1) and (2) of 2.2.2]. 

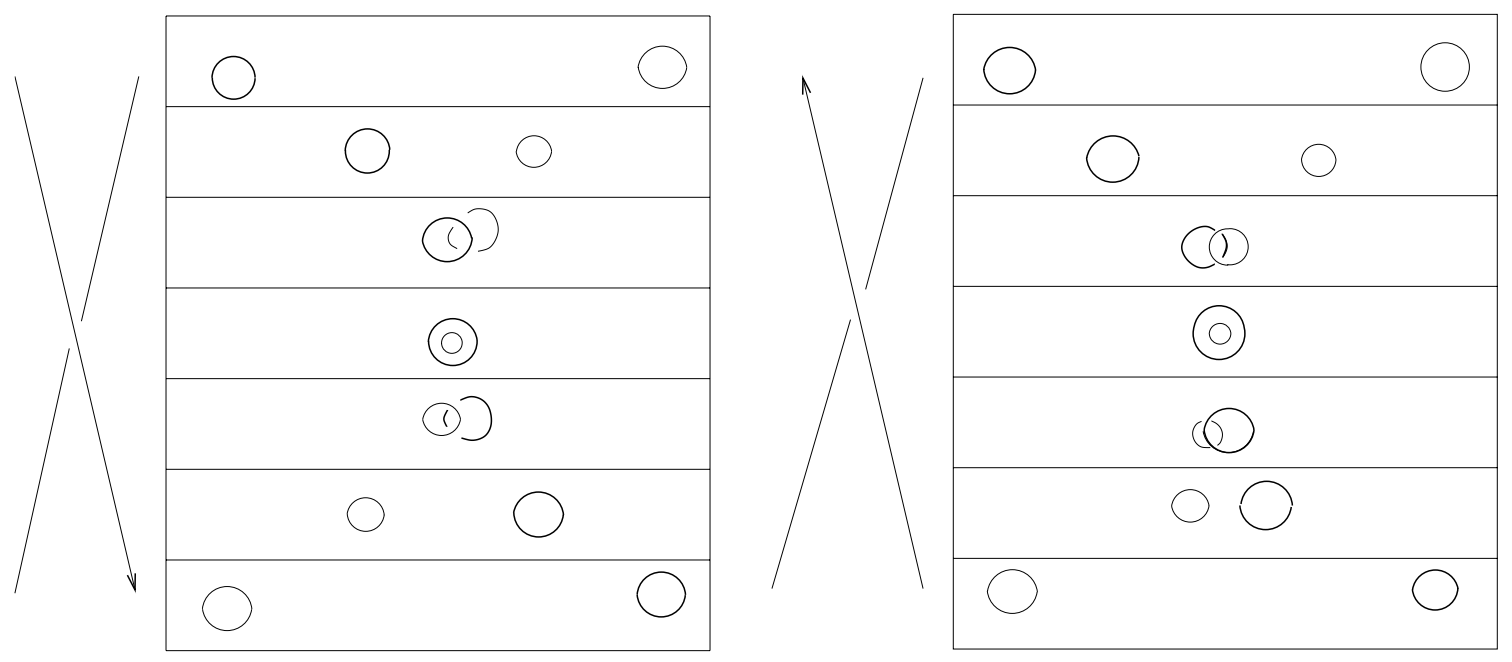

Figure 10. Associating a knotted torus to a virtual knot: classical crossing points, second case. All circles are oriented counterclockwise.
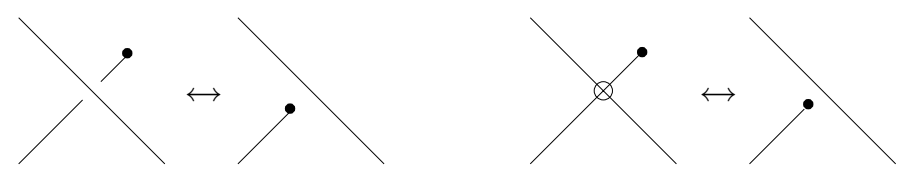

FiguRE 11. Moves on welded virtual arc diagrams.
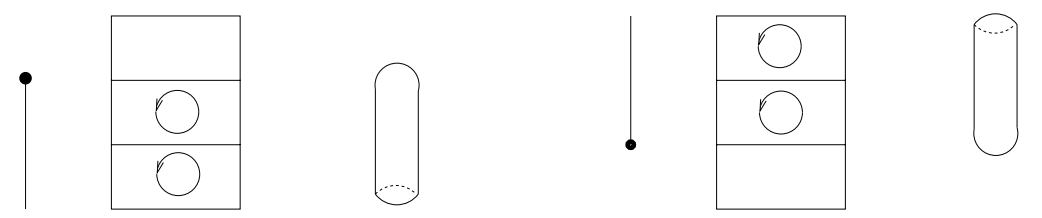

Figure 12. The tube of a welded virtual arc close to the end-points.

2.2.1 Welded virtual arcs. A virtual arc diagram is, by definition, an immersion of a disjoint union of intervals $[0,1]$ into the plane $\mathbb{R}^{2}$, where the 4 -valent vertices of the immersion can represent either classical or virtual crossings. The definition of a welded virtual arc is similar to the definition of a welded virtual knot, but considering in addition the moves of Figure 11; see [Sat00].

A sphere link is, by definition, an embedding of a disjoint union of spheres $S^{2}$ into $S^{4}$, considered up to ambient isotopy. Similarly to ribbon torus links in $S^{4}$, any ribbon sphere link admits a presentation as the tube $T(A)$, where $A$ is a welded virtual arc. Here $T(A)$ is defined in the same way as the tube of a welded virtual knot, considering additionally the movies of Figure 12 at the end-points of the $\operatorname{arcs}$ of $A$. Therefore $T(A)$ is an embedding of a disjoint union of spheres $S^{2}$ into $S^{4}$.

Suppose that the arc $A$ is classical, and that it sits inside the semiplane $\{z \geqslant 0\}$ of $\mathbb{R}^{3}$, intersecting the plane $\{z=0\}$ at the end-points of $A$, transversally. Then in fact $T(A)$ is the spun knot of $A$; see [Ros74, Sat00, CKS04].

We can define the knot group of a welded virtual arc exactly in the same way as we defined the combinatorial fundamental group of the complement of a welded virtual knot. As in the case of welded virtual knots, the map $A \mapsto T(A)$ preserves knot groups; see [Sat00]. 


\section{H. Kauffman and J. FARia Martins}
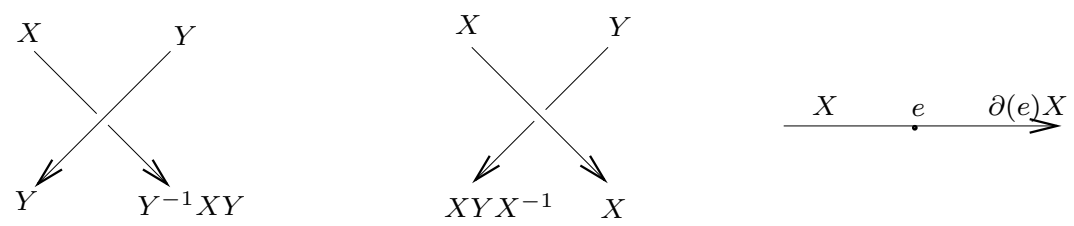

FIGURE 13. Definition of a colouring of a dotted knot diagram.

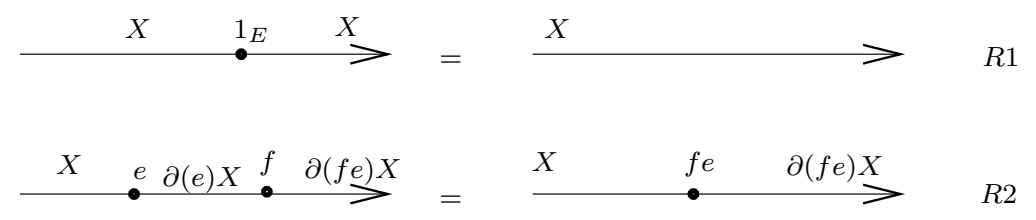

FiguRE 14. Relations on colourings.

Suppose that $A$ is a classical arc (with one component) sitting in the semiplane $\{z \geqslant 0\}$ of $\mathbb{R}^{3}$, intersecting the plane $\{z=0\}$ at the end-points of $A$. Let $K$ be the obvious closure of $A$. Then it is easy to see that $A$ and $K$ have the same knot groups. Note that the fact that $A$ is classical is essential for this to hold. This is also true if $A$ may have some $S^{1}$-components, even though it is strictly necessary that $A$ have only one component homeomorphic to $[0,1]$.

\subsection{Crossed module invariants of knotted surfaces}

A crossed module (see [Bro99]) $\mathcal{G}=(E \stackrel{\partial}{\rightarrow} G, \triangleright)$ is given by a group morphism $\partial: E \rightarrow G$ together with a left action $\triangleright$ of $G$ on $E$ by automorphisms. The conditions on $\partial$ and $\triangleright$ are:

(i) $\partial(X \triangleright e)=X \partial(e) X^{-1}$, for all $X \in G$, for all $e \in E$;

(ii) $\partial(e) \triangleright f=e f e^{-1}$, for all $e, f \in E$.

Note that the second condition implies that the subgroup ker $\partial$ of $E$ is central in $E$, whereas the first implies that ker $\partial$ is $G$-invariant.

A dotted knot diagram is, by definition, a regular projection of a bivalent graph, in other words of a link, possibly with some extra bivalent vertices inserted. Let $D$ be a dotted knot diagram, which we suppose to be oriented. Let also $\mathcal{G}=(E \stackrel{\partial}{\rightarrow} G, \triangleright)$ be a finite crossed module.

Definition 2.1. A colouring of $D$ is an assignment of an element of $G$ to each arc of $D$ and of an element of $E$ to each bivalent vertex of $D$ satisfying the conditions of Figure 13.

Definition 2.2. Let $D$ be a knot diagram (without vertices). A dotting of $D$ is an insertion of bivalent vertices in $D$, considered up to a planar isotopy sending $D$ to $D$, setwise. If $D$ is an oriented knot diagram, let $V(D)$ be the free $\mathbb{Q}$-vector space on the set of all colourings of all dottings of $D$.

Consider now the relations of Figures 14 and 15. It is straightforward to see that they are local on the knot diagrams and that they transform colourings into colourings.

DeFinition 2.3. Let $D$ be an oriented knot diagram (without vertices). The vector space $\mathcal{V}(D)$ is defined as the vector space obtained from $V(D)$ by modding out by the relations $R 1$ to $R 6$.

Let $D$ and $D^{\prime}$ be oriented knot diagrams. If $D$ and $D^{\prime}$ differ by planar isotopy, then there exists an obvious map $\mathcal{V}(D) \rightarrow \mathcal{V}\left(D^{\prime}\right)$. In fact, if $D$ and $D^{\prime}$ differ by a Reidemeister move or a Morse move (in other words a birth/death of a circle or a saddle point), then there also exists a well defined 
INVARIANTS OF WELDED VIRTUAL KNOTS
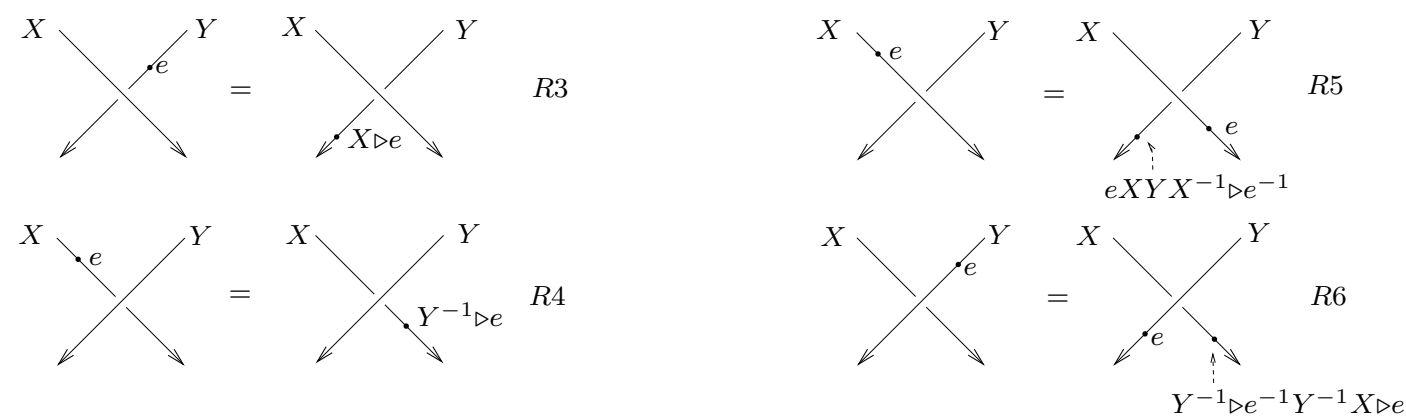

Figure 15. Relations on colourings.
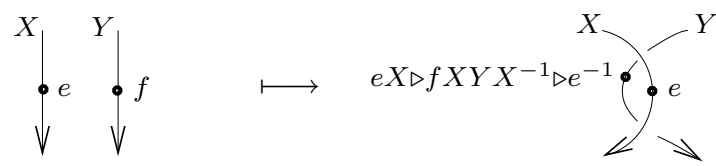

Figure 16. Map assigned to positive Reidemeister-II move.
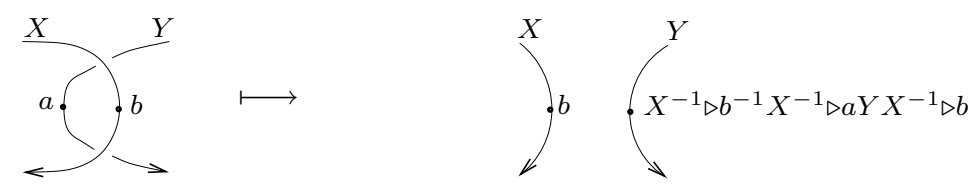

FiguRE 17. Map assigned to negative Reidemeister-II move.
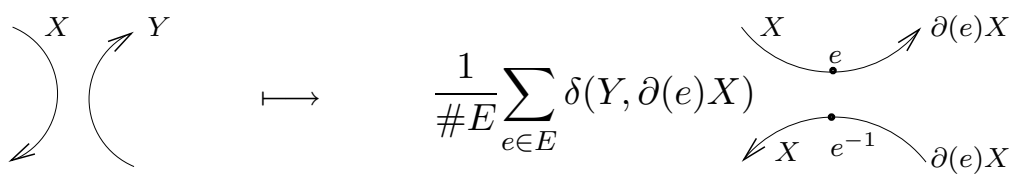

FiguRE 18. Map associated to saddle point moves.

1
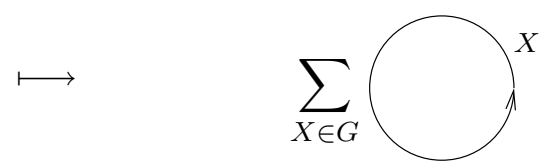

FiguRE 19. Map associated with births of a circle.

map $\mathcal{V}(D) \rightarrow \mathcal{V}\left(D^{\prime}\right)$. All this is explained in [FM07a]. In Figures 16, 17, 18, 19 and 20 we display the definition of these maps for the case of the Reidemeister-II move and the Morse moves, which we are going to need in this paper. The remaining cases of these moves can be dealt with by doing the transition shown in Figure 21, and using the relations $R 1$ to $R 6$. In Figure 18, $\delta$ is a Kronecker delta.

Therefore, any movie of an oriented knotted surface $\Sigma$ can be evaluated to give an element $I_{\mathcal{G}}(\Sigma) \in \mathbb{Q}$.

Theorem 2.4. The evaluation $I_{\mathcal{G}}$ of a movie of an oriented knotted surface defines an isotopy invariant of oriented knotted surfaces. 


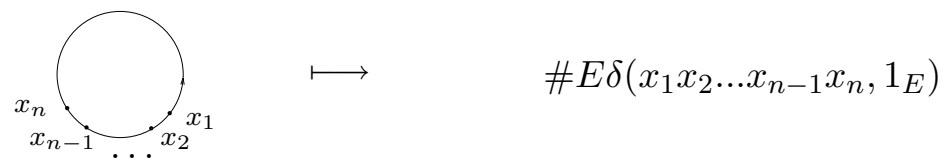

Figure 20. Map associated with deaths of a circle.

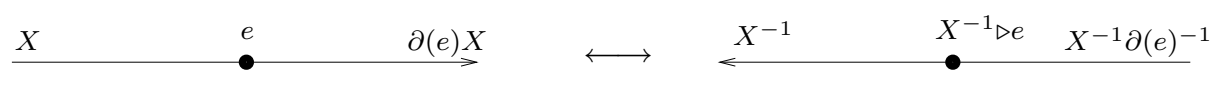

FiguRE 21. Inversion of strands.
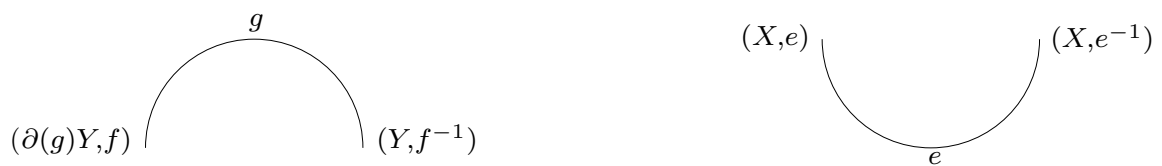

FiguRE 22. Relations at maximal and minimal points.

This is shown in [FM07a]. The homotopy theoretical interpretation of the isotopy invariant $I_{\mathcal{G}}$ is discussed in [FM08, FM07b, FMP07]. The construction of the invariant $I_{\mathcal{G}}$ was initially inspired by Yetter's invariant of manifolds; see [Yet93, Por96, Por98].

Actually $I_{\mathcal{G}}$ defines an embedded Topological Quantum Field Theory, in other words, an invariant of link cobordisms considered up to ambient isotopy fixing both ends.

2.3.1 The case of ribbon knotted torus. As we have seen, if $\Sigma$ is a ribbon knotted surface, which topologically is the disjoint union of tori $S^{1} \times S^{1}$ or spheres $S^{2}$, then we can represent it as the tube $T(K)$ of a welded virtual knot $K$, in the first case, or the tube $T(A)$ of a welded virtual arc $A$, in the second case.

We want to find an algorithm for calculating $I_{\mathcal{G}}(T(K))$, where $K$ is a welded virtual knot, directly from a diagram of $K$ itself, and analogously for a welded virtual arc $A$. A careful look at the definition of the invariant $I_{\mathcal{G}}$ together with the definition of the tube map in $\S 2.2$ leads to the following definition.

Definition 2.5. Let $\mathcal{G}=(E \stackrel{\partial}{\rightarrow} G, \triangleright)$ be a crossed module. Let also $D$ be a welded virtual knot diagram. Suppose that the projection on the second variable defines a Morse function on $D$. A $\mathcal{G}$-colouring ${ }^{2}$ of $D$ is an assignment of a pair $(X, f)$, where $X \in G$ and $f \in \operatorname{ker} \partial$, to each connected component of $D$ minus its set of crossings and extreme points; of an element $e \in$ ker $\partial$ to each minimal point; and of an element $g \in E$ to each maximal point, satisfying the conditions shown in Figures 22 and 23.

The reason for considering these relations is obvious from Figures 24 and 25 and their counterparts for different types of crossings. Note that ker $\partial \subset E$ is central in $E$. However, for this calculus to approximate the definition of $I_{\mathcal{G}}(T(D))$, for $D$ a virtual knot diagram, the relation of Figure 26

${ }^{2}$ This should not be confused with the notion of a colouring which was considered in the definition of the invariant $I_{\mathcal{G}}$, above. 


\section{INVARIANTS OF WELDED VIRTUAL KNOTS}
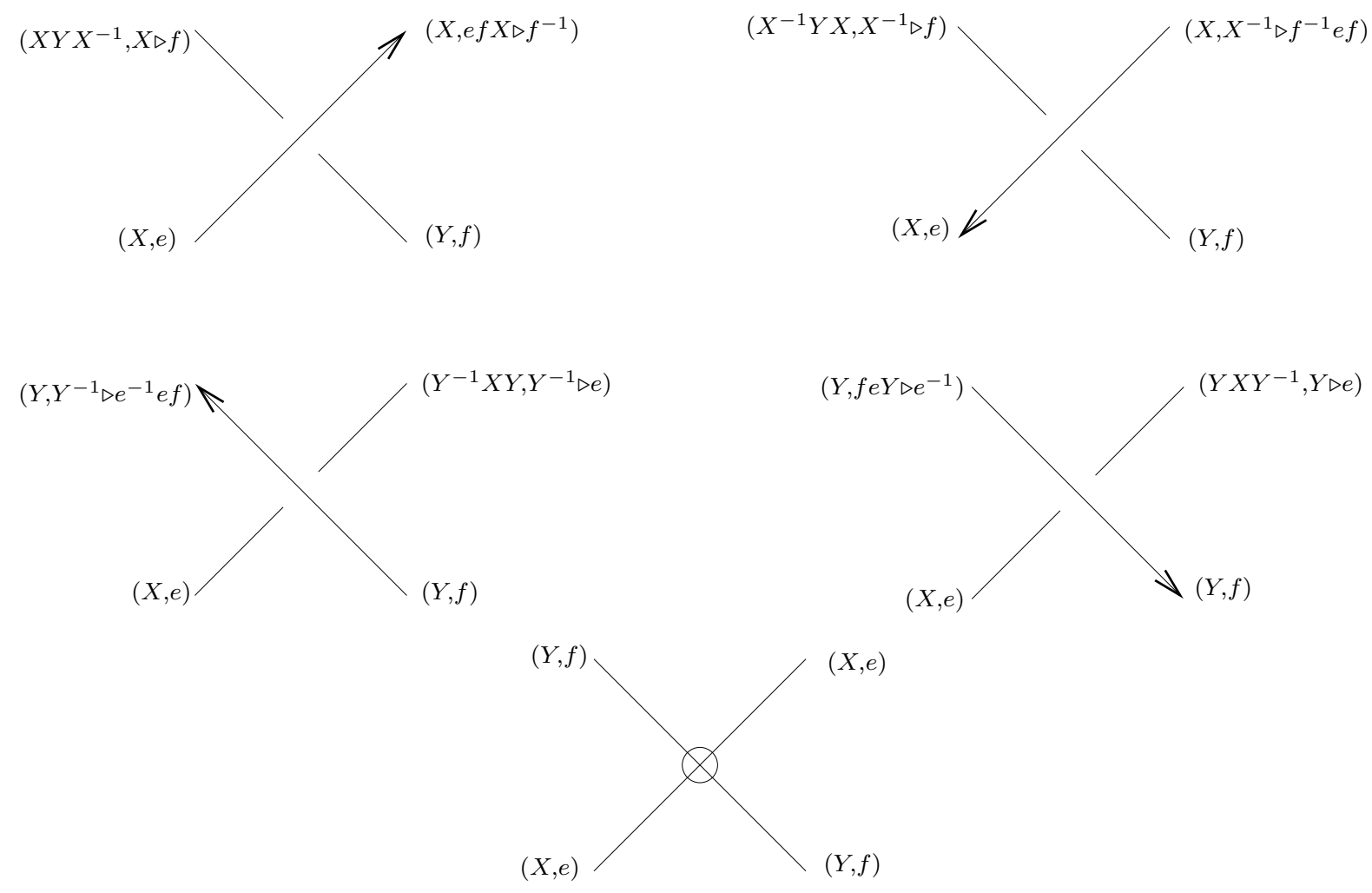

FiguRE 23. Relations at crossings.

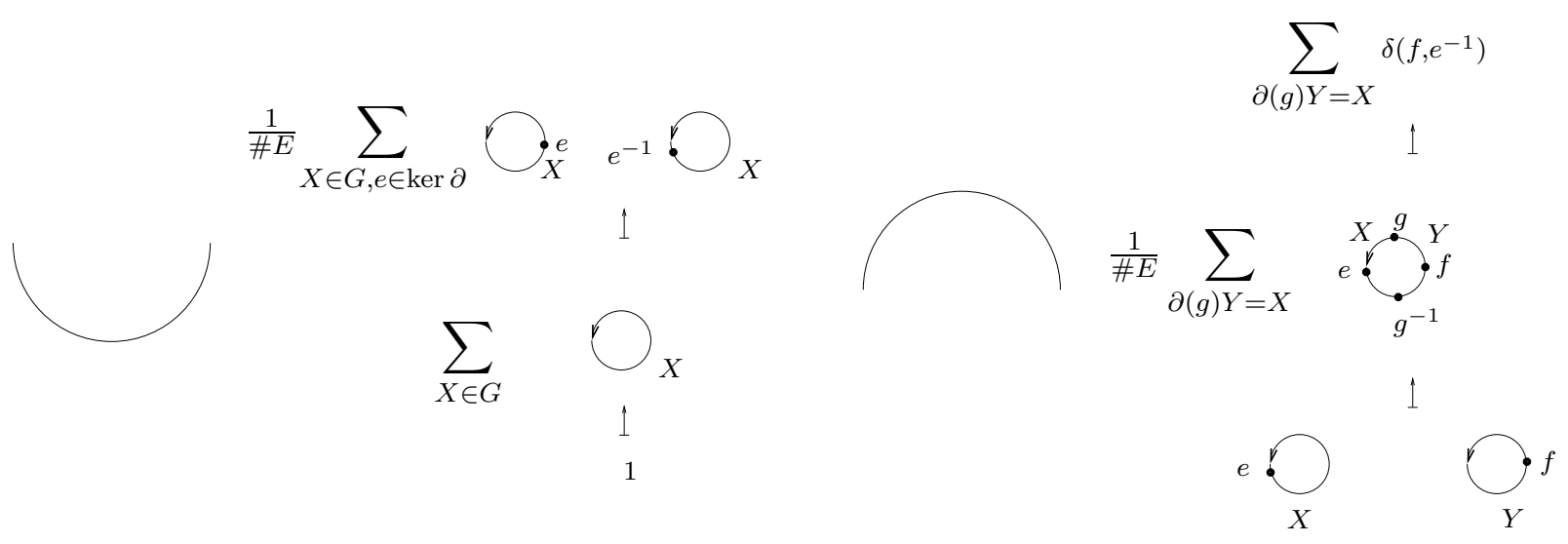

Figure 24. Calculation of $I_{\mathcal{G}}$ of the tube of a welded virtual knot: minimal and maximal points.

still needs to be incorporated into the calculations. To avoid needing to involve this relation, we consider the following restriction on the crossed modules with which we work.

Definition 2.6 (Automorphic crossed module). A crossed module $\mathcal{G}=(E \stackrel{\partial}{\rightarrow} G, \triangleright)$ is called automorphic if $\partial(e)=1$, for all $e \in E$. Therefore, an automorphic crossed module is given simply by two groups $G$ and $E$, with $E$ abelian, and a left action $\triangleright$ of $G$ on $E$ by automorphisms.

Definition 2.7 (Reduced $\mathcal{G}$-colourings). Let $\mathcal{G}=(E, G, \triangleright)$ be an automorphic crossed module. Let also $D \subset \mathbb{R}^{2}$ be a virtual knot diagram, such that the projection on the second variable is a Morse function on $D$. A reduced $\mathcal{G}$-colouring of $D$ is given by an assignment of a pair $(X, e) \in G \times E$ to each 
L. H. Kauffman and J. FARia Martins<smiles>[Y][Y]1[Y]=[Y9]([Y])CC1</smiles>

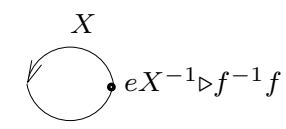

1
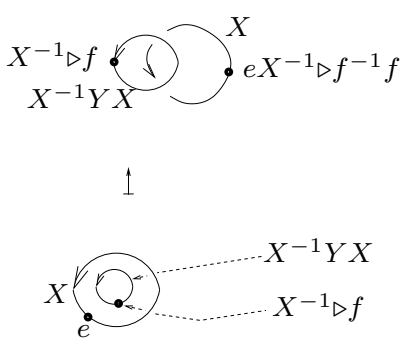

1

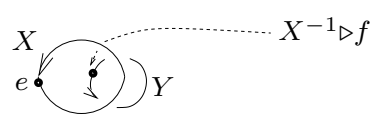

$\bigoplus_{e} X$

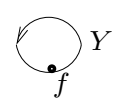

Figure 25. Calculation of $I_{\mathcal{G}}$ of the tube $T(D)$ of a welded virtual knot $D$ : the type of crossings relative to Figure 9.

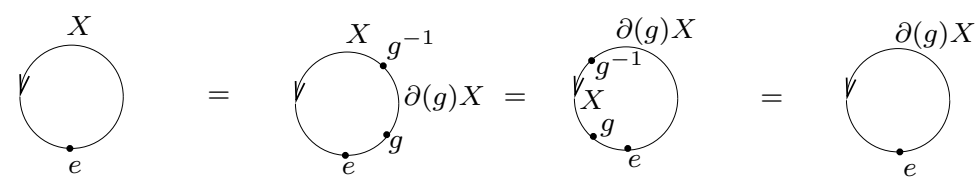

Figure 26. An identity. Here $e \in \operatorname{ker} \partial$.
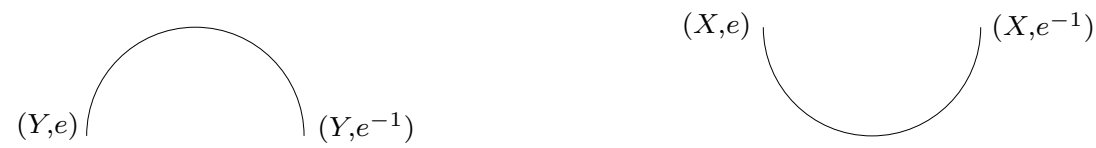

FIGURE 27. Reduced $\mathcal{G}$-colouring at extreme points.

connected component of $D$ minus its set of crossings and extreme points, satisfying the relations of Figures 23 and 27.

The following result is easy to prove by using all the information we provided, and the fact that, for any knot diagram, the number of minimal points of it equals the number of maximal points.

Theorem 2.8. Let $D$ be a virtual knot diagram, such that the projection on the second variable is a Morse function on $D$. Let also $\mathcal{G}=(E, G, \triangleright)$ be a finite automorphic crossed module. Consider the quantity

$$
\mathcal{H}_{\mathcal{G}}(D)=\#\{\text { reduced } \mathcal{G} \text {-colourings of } D\}
$$



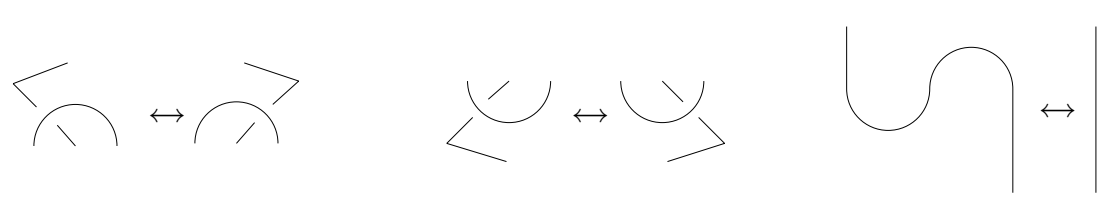

Figure 28. Sample of Yetter's moves capturing planar isotopy.

Then $\mathcal{H}_{\mathcal{G}}(D)$ is an invariant of welded virtual knots. In fact

$$
\mathcal{H}_{\mathcal{G}}(D)=I_{\mathcal{G}}(T(D)) \text {. }
$$

Here $I_{\mathcal{G}}$ is the crossed module invariant of oriented knotted surfaces defined in [FM07a].

Exercise 1. Check directly that $\mathcal{H}_{\mathcal{G}}$ (where $\mathcal{G}$ is an automorphic finite crossed module) is an invariant of welded virtual knots. Note that, together with the moves defining welded virtual knots, we still need to check invariance under planar isotopy, thus forcing us to check invariance under the moves of the type depicted in Figure 28, usually called Yetter's moves; see [Yet89, FY89]. It is important to note that we need to consider all the possible different crossing information, and, since we are working in the oriented case, all the possible orientations of the strands.

Let $\mathcal{G}=(E \stackrel{\partial}{\rightarrow} G, \triangleright)$ be a crossed module. Define $\pi_{1}(\mathcal{G})=\operatorname{coker}(\partial)$ and $\pi_{2}(\mathcal{G})=$ ker $\partial$, which is an abelian group. Then $\pi_{1}(\mathcal{G})$ has a natural left action $\triangleright^{\prime}$ on $\pi_{2}(\mathcal{G})$ by automorphisms. In particular $\Pi(\mathcal{G})=\left(\pi_{2}(\mathcal{G}), \pi_{1}(\mathcal{G}), \triangleright^{\prime}\right)$ is an automorphic crossed module. In fact $\mathcal{G}$ also determines a cohomology class $k^{3} \in H^{3}\left(\pi_{1}(\mathcal{G}), \pi_{2}(\mathcal{G})\right)$, called the $k$-invariant of $\mathcal{G}$.

It is not difficult to extend the invariant $\mathcal{H}_{\mathcal{G}}(D)$, where $D$ is a welded virtual knot, to handle non-automorphic crossed modules $\mathcal{G}$, so that $\mathcal{H}_{\mathcal{G}}(D)=I_{\mathcal{G}}(T(D))$. We do this by incorporating the relation in Figure 26 into the notion of a $\mathcal{G}$-colouring of a virtual knot diagram. However, it is possible to prove that, for any welded virtual knot $D$ and any finite crossed module $\mathcal{G}$, we have that $I_{\mathcal{G}}(T(D))$ equals $I_{\Pi(\mathcal{G})}(T(D))$, apart from normalisation factors. This can be proved by using the graphical framework presented in this paper. Hence, we do not lose generality if we restrict our attention only to automorphic crossed modules.

Problem 1. Let $\mathcal{G}=(E, G, \triangleright)$ be an automorphic crossed module. Find a ribbon Hopf algebra $\mathcal{A}_{\mathcal{G}}$ acting on the vector space freely generated by $G \times E$ such that $\mathcal{H}_{\mathcal{G}}$ is the Reshetikhin-Turaev invariant of knots associated to it (see [RT90]), and so that the case of welded virtual knots also follows from this Hopf algebra framework in a natural way. Note that, in the case when $E=0$, we can take $\mathcal{A}_{\mathcal{G}}$ to be the quantum double of the function algebra on $G$. The solution to this problem would be somehow the quantum double of a finite categorical group, and therefore would be of considerable importance.

2.3.2 The case of welded virtual arcs. Let $\mathcal{G}=(E, G, \triangleright)$ be a finite automorphic crossed module. Let also $A$ be a virtual arc diagram. The notion of a reduced $\mathcal{G}$-colouring of $A$ is totally analogous to the concept of a reduced $\mathcal{G}$-colouring of a virtual knot diagram, considering that if an arc of $A$ has a free end then it must be coloured by $\left(X, 1_{E}\right)$, where $X \in G$; see Figure 29. One can see this from Figure 12. We have the following result.

Theorem 2.9. Let $A$ be a virtual arc diagram. The quantity

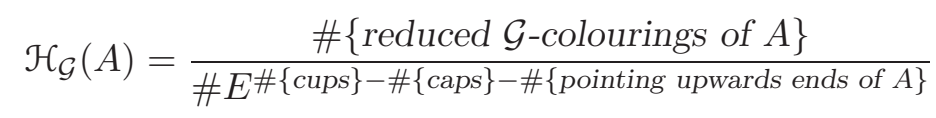

is an invariant of $A$ as a welded virtual arc. In fact

$$
\mathcal{H}_{\mathcal{G}}(A)=I_{\mathcal{G}}(T(A))
$$


L. H. Kauffman and J. Faria Martins

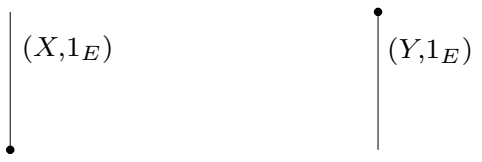

FiguRE 29. Reduced $\mathcal{G}$-colourings of welded virtual arcs at end-points. Here $X, Y \in G$.
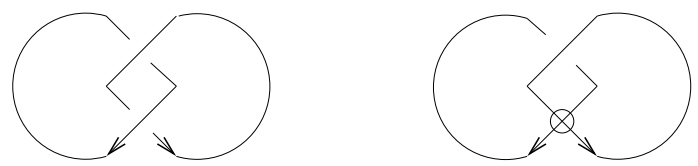

Figure 30. Classical and virtual Hopf links.

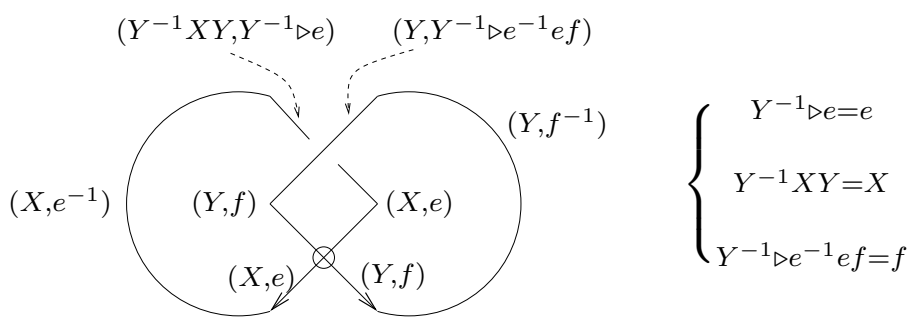

Figure 31. Calculation of the crossed module invariant of the virtual Hopf link $L$.

Therefore, the graphical framework presented in this paper is also a calculational device for calculating the crossed module invariant of spun knots, according to $§ 2.2 .1$.

The invariant $\mathcal{H}_{\mathcal{G}}$ of Theorem 2.9 actually is an invariant of virtual arcs of which some components may be circles. In fact, it also naturally extends to an invariant of welded virtual graphs, to be defined in $\S 3.5 .2$.

\section{Examples}

\subsection{Virtual and classical Hopf link}

3.1.1 Virtual Hopf link. The simplest non-trivial welded virtual link is the virtual Hopf link $L$, depicted in Figure 30. Note that $L$ is linked since its knot group is $\{X, Y: X Y=Y X\} \cong \mathbb{Z}^{2}$.

Let $\mathcal{G}=(E, G, \triangleright)$ be a finite automorphic crossed module. Let us calculate the crossed module invariant $\mathcal{H}_{\mathcal{G}}$ of the virtual Hopf link $L$. This calculation appears in Figure 31. From this we can conclude that

$$
\begin{aligned}
\mathcal{H}_{\mathcal{G}}(L) & =\#\left\{X, Y \in G ; e, f \in E \mid X Y=Y X, Y^{-1} \triangleright e=e\right\} \\
& =\# E \#\left\{X, Y \in G ; e \in E \mid X Y=Y X, Y^{-1} \triangleright e=e\right\} .
\end{aligned}
$$

Note that the previous equation simplifies to

$$
\mathcal{H}_{\mathcal{G}}(L)=\# E \# G \#\left\{Y \in G ; e \in E \mid Y^{-1} \triangleright e=e\right\},
$$

when the group $G$ is abelian. On the other hand it is easy to see that if $O^{2}$ is a pair of unlinked unknots then we have

$$
\mathcal{H}_{\mathcal{G}}\left(O^{2}\right)=\# G^{2} \# E^{2} .
$$

From (2) and (3), it thus follows that any finite automorphic crossed module $(E, G, \triangleright)$ with $G$ abelian 
INVARIANTS OF WELDED VIRTUAL KNOTS

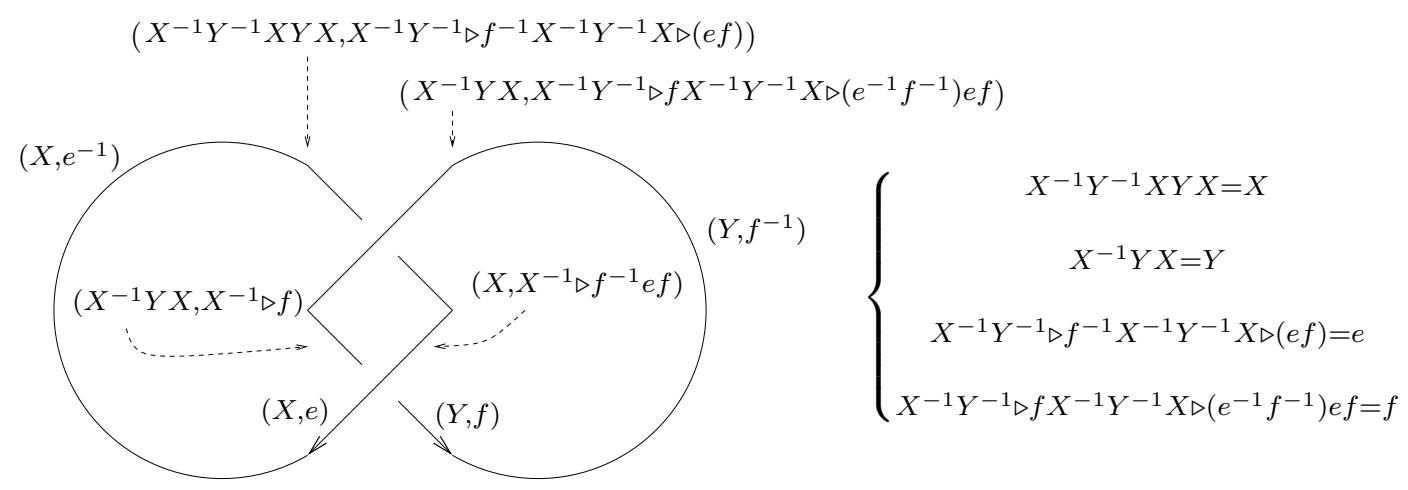

FiguRE 32. Calculation of the crossed module invariant of the Hopf link.

sees the knotting of the virtual Hopf link if there exist $Y \in G$ and $e \in E$ such that $Y^{-1} \triangleright e \neq e$. This is verified in any automorphic crossed module $(E, G, \triangleright)$ with $\triangleright$ being a non-trivial action of $G$ on $E$.

Consider the automorphic crossed module $\mathcal{A}=\left(E=\mathbb{Z}_{3}, G=\mathbb{Z}_{2}, \triangleright\right)$ such that $1 \triangleright a=a$ and $-1 \triangleright a=-a$, where $a \in \mathbb{Z}_{3}$ and $\mathbb{Z}_{2}=(\{1,-1\}, \times)$; see [BM06]. Then this crossed module detects the knottedness of the virtual Hopf link $L$. If fact $\mathcal{H}_{\mathcal{A}}(L)=6 \#\left\{Y \in \mathbb{Z}_{2} ; e \in \mathbb{Z}_{3} \mid Y^{-1} \triangleright e=e\right\}=24$, whereas $\mathcal{H}_{\mathcal{A}}\left(O^{2}\right)=36$.

3.1.2 The Hopf link. The Hopf link $H$ is depicted in Figure 30. Note that the fundamental group of the complement of it is, similarly with the virtual Hopf link $L$, isomorphic with $\mathbb{Z}^{2}$.

Let us calculate the crossed module invariant of the Hopf link $H$. To this end, let $\mathcal{G}=(E, G, \triangleright)$ be a finite automorphic crossed module. We display the calculation of $\mathcal{H}_{\mathcal{G}}(H)$ in Figure 32. This permits us to conclude that

$$
\mathcal{H}_{\mathcal{G}}(H)=\#\left\{X, Y \in G ; e, f \in E \mid \begin{array}{c}
X Y=Y X \\
X^{-1} Y^{-1} \triangleright f^{-1} X^{-1} Y^{-1} X \triangleright(e f)=e
\end{array}\right\},
$$

which particularises to

$$
\mathcal{H}_{\mathcal{G}}(H)=\#\left\{X, Y \in G ; e, f \in E: X^{-1} Y^{-1} \triangleright f^{-1} Y^{-1} \triangleright(e f)=e\right\},
$$

in the case when $G$ is abelian. This is in agreement with the calculation in [FM08].

Let us see that the Hopf link $H$ is not equivalent to the virtual Hopf link $L$ as a welded virtual link. Consider the automorphic crossed module $\mathcal{A}=\left(E=\mathbb{Z}^{3}, G=\mathbb{Z}^{2}, \triangleright\right)$ defined above. We have (note that we switch to additive notation, more adapted to this example)

$$
\begin{aligned}
\mathcal{H}_{\mathcal{A}}(H) & =\#\left\{X, Y \in \mathbb{Z}_{2} ; e, f \in \mathbb{Z}_{3}:-X Y \triangleright f+Y \triangleright(e+f)=e\right\} \\
& =\#\left\{X, Y \in \mathbb{Z}_{2} ; e, f \in \mathbb{Z}_{3}:-X Y \triangleright f+Y \triangleright f=e-Y \triangleright e\right\} .
\end{aligned}
$$

In the case $Y=1$, we are led to the equation $-X \triangleright f+f=0$, which has $4 \times 3$ solutions in $\mathbb{Z}_{2} \times \mathbb{Z}_{3} \times \mathbb{Z}_{3}$. In the case $Y=-1$, we get the equation $e=2^{-1}(X \triangleright f-f)$, which has $3 \times 2$ solutions in $\mathbb{Z}_{2} \times \mathbb{Z}_{3} \times \mathbb{Z}_{3}$. Therefore, we obtain $\mathcal{H}_{\mathcal{A}}(H)=18$.

Therefore, we have proved that the virtual Hopf link is not equivalent to the Hopf link as a welded virtual link, and also that the Hopf link is knotted, by using the crossed module invariant.

As we have referred to before, the knot groups of the Hopf link and the virtual Hopf link are both isomorphic with $\mathbb{Z}^{2}$. Therefore, we have proved that the crossed module invariant $\mathcal{H}_{\mathcal{G}}$ sees beyond the fundamental group of the complement of a welded virtual knot. 


\section{H. Kauffman and J. FARia Martins}
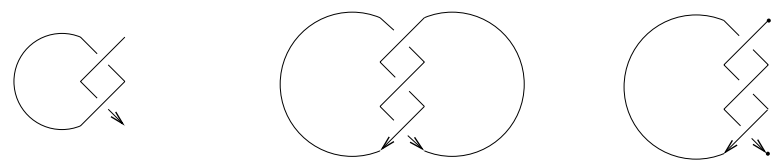

Figure 33. The Hopf arc $H A$, the trefoil knot $3_{1}$ and the trefoil arc $3_{1}^{\prime}$.

Since the correspondence $K \mapsto T(K)$, where $K$ is a welded virtual link, preserves the fundamental groups of the complement we have also proved the following result.

TheOREM 3.1. The crossed module invariant $I_{\mathcal{G}}$ of knotted surfaces defined in [FM07a, FM08] is powerful enough to distinguish between knotted surfaces $\Sigma, \Sigma^{\prime} \subset S^{4}$, with $\Sigma$ diffeomorphic with $\Sigma^{\prime}$, whose complements have isomorphic fundamental groups, at least in a particular case.

Therefore, one of the main open problems about the crossed module invariant $I_{\mathcal{G}}$ of knotted surfaces that prevails is whether the invariant $I_{\mathcal{G}}$ can distinguish between knotted surfaces whose complements have isomorphic fundamental groups and second homotopy groups, seen as $\pi_{1}$-modules, but have distinct Postnikov invariants $k^{3} \in H^{3}\left(\pi_{1}, \pi_{2}\right)$. This problem was referred to in [FM08]. Examples of pairs of knotted surfaces like this do exist; see [PS85].

Exercise 2. Consider the Hopf arc $H A$ depicted in Figure 33. Prove that $\mathcal{H}_{\mathcal{G}}(H A)=\mathcal{H}_{\mathcal{G}}(L)$, where $L$ is the virtual Hopf link. Here $\mathcal{G}=(E, G, \triangleright)$ is any finite automorphic crossed module. In fact, cf. $\S 3.5 .1, T(L)$ is obtained from $T(H A)$ by adding a trivial 1-handle, which explains this identity. We will go back to this later in $\S 3.5 .2$.

\subsection{Trefoil knot and trefoil arc}

The trefoil knot $3_{1}$ and the trefoil arc $3_{1}^{\prime}$ are depicted in Figure 33.

Let us calculate the crossed module invariant of the trefoil knot $3_{1}$. Let $\mathcal{G}=(E, G, \triangleright)$ be a finite automorphic crossed module. The calculation of $\mathcal{H}_{\mathcal{G}}\left(3_{1}\right)$ appears in Figure 34 . This permits us to conclude that

$$
\begin{aligned}
\mathcal{H}_{\mathcal{G}}\left(3_{1}\right) & =\#\left\{X, Y \in G ; e, f \in E \mid \begin{array}{c}
X^{-1} Y^{-1} X^{-1}=Y^{-1} X^{-1} Y^{-1} \\
Y^{-1} X^{-1} Y^{-1} \triangleright f Y^{-1} X^{-1} Y^{-1} X \triangleright(e f)^{-1} Y^{-1} \triangleright(e f)=e
\end{array}\right\} \\
& =\#\left\{X, Y \in G ; e, f \in E \mid \begin{array}{c}
X^{-1} Y^{-1} X^{-1}=Y^{-1} X^{-1} Y^{-1} \\
Y^{-1} X^{-1} Y^{-1} \triangleright f X^{-1} Y^{-1} \triangleright(e f)^{-1} Y^{-1} \triangleright(e f)=e
\end{array}\right\} .
\end{aligned}
$$

This simplifies to

$$
\mathcal{H}_{\mathcal{G}}\left(3_{1}\right)=\#\left\{X \in G ; e, f \in E \mid X^{-3} \triangleright f X^{-2} \triangleright(e f)^{-1} X^{-1} \triangleright(e f)=e\right\},
$$

when $\mathcal{G}=(E, G, \triangleright)$ is an automorphic crossed module with $G$ abelian; see $\S 3.3 .2$.

Note that the crossed module invariant of the trefoil arc $3_{1}^{\prime}$ can also be obtained from this calculation, by making $f=1_{E}$, and inserting the necessary normalisation factors; see $\S 2.3 .2$. This yields

which simplifies to

$$
\mathcal{H}_{\mathcal{G}}\left(3_{1}^{\prime}\right)=\# E \#\left\{\begin{array}{l|l}
X, Y \in G ; e \in E & \begin{array}{c}
X^{-1} Y^{-1} X^{-1}=Y^{-1} X^{-1} Y^{-1} \\
X^{-1} Y^{-1} \triangleright e^{-1} Y^{-1} \triangleright e=e
\end{array}
\end{array}\right\},
$$

$$
\mathcal{H}_{\mathcal{G}}\left(3_{1}^{\prime}\right)=\# E \#\left\{X \in G ; e \in E: X^{-2} \triangleright e^{-1} X^{-1} \triangleright e=e\right\},
$$

whenever $G$ is abelian. This is coherent with the calculation in [FM07a, FM08]. 
INVARIANTS OF WELDED VIRTUAL KNOTS

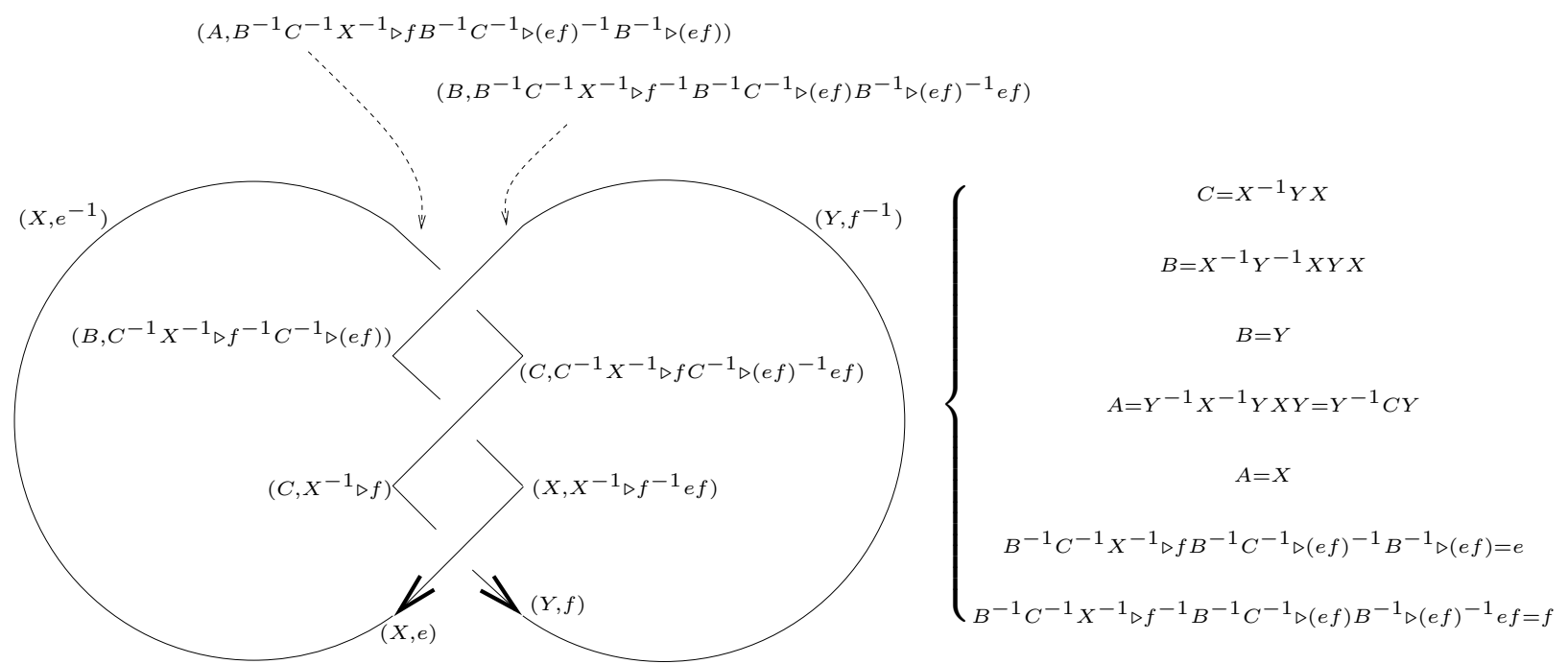

Figure 34. Calculation of the crossed module invariant of the trefoil knot $3_{1}$.

Observe that from (7) and (9) it follows that (we switch to additive notation)

$$
\begin{aligned}
\mathcal{H}_{\mathcal{G}}\left(3_{1}\right) & =\#\left\{X \in G ; e, f \in E: X^{-3} \triangleright f-X^{-2} \triangleright(e+f)+X^{-1} \triangleright(e+f)=e\right\} \\
& =\#\left\{X \in G ; e, f \in E: X^{-2} \triangleright\left(X^{-1} \triangleright f-e\right)-X^{-1} \triangleright\left(X^{-1} \triangleright f-e\right)+\left(X^{-1} \triangleright f-e\right)=0\right\} \\
& =\mathcal{H}_{\mathcal{G}}\left(3_{1}^{\prime}\right) .
\end{aligned}
$$

Thus

$$
\mathcal{H}_{\mathcal{G}}\left(3_{1}\right)=\mathcal{H}_{\mathcal{G}}\left(3_{1}^{\prime}\right)
$$

whenever $\mathcal{G}=(E, G, \triangleright)$ is an automorphic crossed module with $G$ abelian. An analogous identity holds for any classical one-component knot; see $\S 3.3 .2$.

We will consider the crossed module invariants of the trefoil knot and the trefoil arc for the case when $\mathcal{G}=(E, G, \triangleright)$ is an automorphic crossed module with $G$ being a non-abelian group in $\S 3.5 .5$. In this case the previous identity does not hold.

Let us see that $\mathcal{H}_{\mathcal{G}}$ detects the knottedness of the trefoil knot $3_{1}$. The crossed module $\mathcal{A}=$ $\left(\mathbb{Z}_{3}, \mathbb{Z}_{2}, \triangleright\right)$ defined previously detects it. In fact it is easy to see that $\mathcal{H}_{\mathcal{A}}\left(3_{1}\right)=12$. On the other hand, if $O$ is the unknot, we have that $\mathcal{H}_{\mathcal{G}}(O)=\# E \# G$, for any automorphic crossed module $\mathcal{G}=(E, G, \triangleright)$. Thus $3_{1}$ is knotted. Analogously we can prove that the trefoil arc $3_{1}^{\prime}$ is knotted.

Exercise 3. Consider the virtual arc $A$ of Figure 35. Prove that if $\mathcal{G}=(E, G, \triangleright)$ is an automorphic finite crossed module with $G$ abelian then

$$
\mathcal{H}_{\mathcal{G}}(A)=\# E \#\left\{X \in G ; e \in E \mid X^{-2} \triangleright e^{-1} X^{-1} \triangleright e e^{-1}=1\right\} .
$$

Thus the crossed module $\mathcal{A}=\left(\mathbb{Z}_{3}, \mathbb{Z}_{2}, \triangleright\right)$ defined previously detects that it is knotted. However, it is easy to show that the closure of $A$ is the trivial welded virtual knot, a fact confirmed by the crossed module invariant.

\subsection{Universal module constructions}

Let $G$ be an abelian group. Suppose that $\mathcal{G}=(E, G, \triangleright)$ is an automorphic crossed module, where $E$ is an abelian group. Consider a welded virtual link $K$. Suppose that $K$ has $n$ components $S^{1}$, where $n$ is a positive integer. Let $\kappa_{n}=\mathbb{Z}\left[X_{1}, X_{1}^{-1}, \ldots, X_{n}, X_{n}^{-1}\right]$ be the ring of Laurent polynomials 


\section{H. KaufFman And J. FARia Martins}

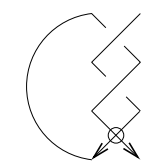

FIgURE 35. A non-trivial welded virtual arc whose closure is trivial.

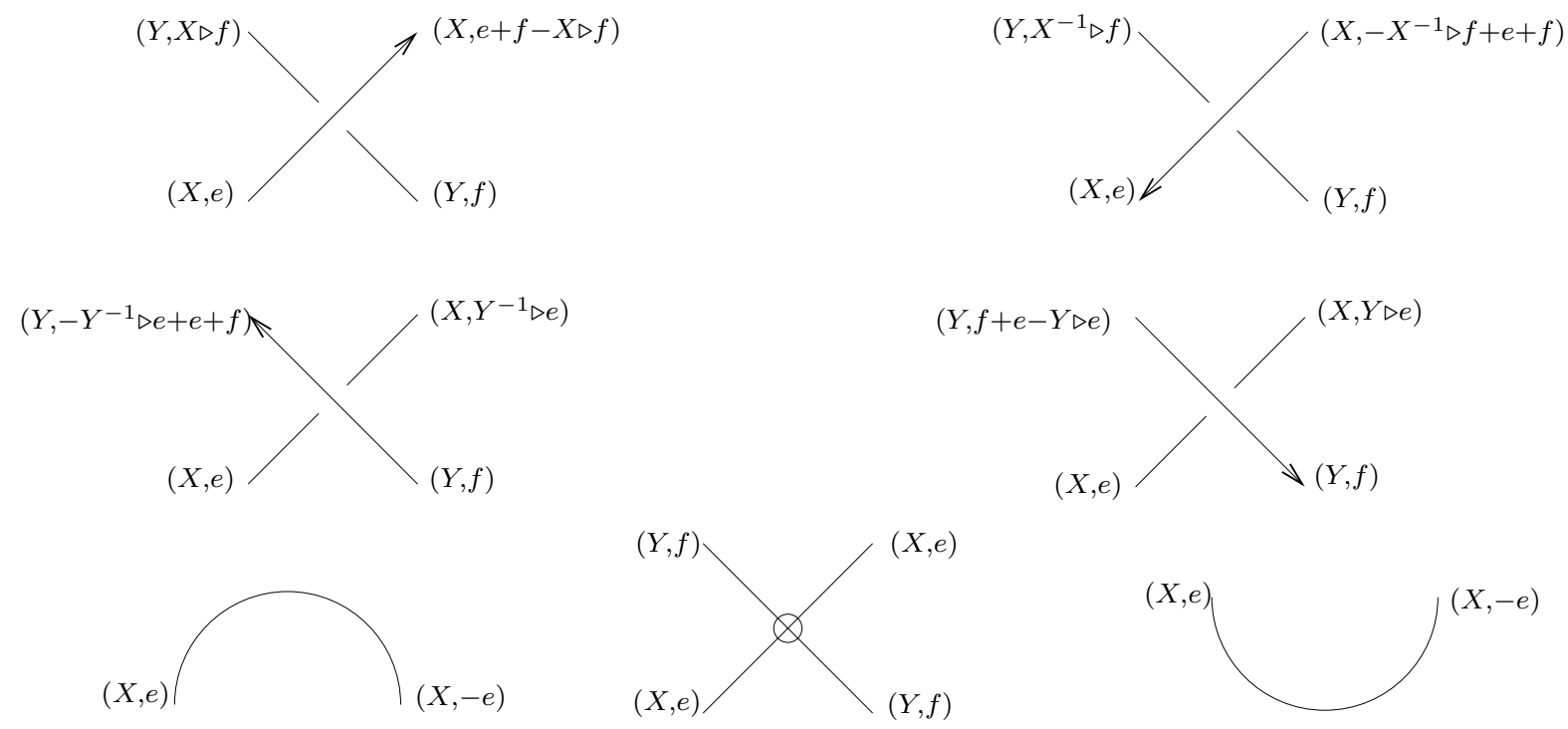

Figure 36. Defining relations for the module $\mathrm{CM}(K)$.

on the formal variables $X_{1}, \ldots X_{n}$. We can assign to $K$ a $\kappa_{n}$-module $\operatorname{CM}(K)$, so that $\mathcal{H}_{\mathcal{G}}(K)$ will satisfy

$$
\mathcal{H}_{\mathcal{G}}(K)=\# \operatorname{Hom}(\mathrm{CM}(K), \mathcal{G}),
$$

where $\operatorname{Hom}(\mathrm{CM}(K), \mathcal{G})$ denotes the set of all crossed module morphisms $\mathrm{CM}(K) \rightarrow \mathcal{G}$.

\subsubsection{The definition of the module $\mathrm{CM}(K)$. This is given as follows.}

Definition 3.2. Let $K$ be a welded virtual link diagram. Suppose that $K$ is an immersion of a disjoint union of $n$ circles $S^{1}$ into the plane, each of which is assigned a variable $X_{i}$, where $i \in\{1, \ldots, n\}$; in other words, suppose that we have a total order on the set of all $S^{1}$-components of $K$. The module $\mathrm{CM}(K)$ is defined as the $\kappa_{n}$-module generated by all the connected components of $K$ minus the set of crossings of $K$ and extreme points of $K$, modding out by the relations of Figure 36 . It is understood that any connected component is assigned a pair $(X, e)$, where $e \in \mathrm{CM}(K)$ is the module element that the connected components define, whereas $X \in\left\{X_{1}, \ldots, X_{n}\right\}$ is the labelling of the $S^{1}$-component of $K$ in which the connected component is included.

By using the same technique as in Exercise 1 we can prove the following result.

Theorem 3.3. Let $K$ be a welded virtual link diagram with $n S^{1}$-components. The isomorphism class of the $\kappa_{n}$-module $\mathrm{CM}(K)$ depends only on the welded virtual link determined by $K$, up to reordering of the $S^{1}$-components of $K$. In addition, if $\mathcal{G}=(E, G, \triangleright)$ is an automorphic finite crossed module with $G$ abelian, we have

$$
\mathcal{H}_{\mathcal{G}}(K)=\# \operatorname{Hom}(\mathrm{CM}(K), \mathcal{G})
$$


INVARIANTS OF WELDED VIRTUAL KNOTS
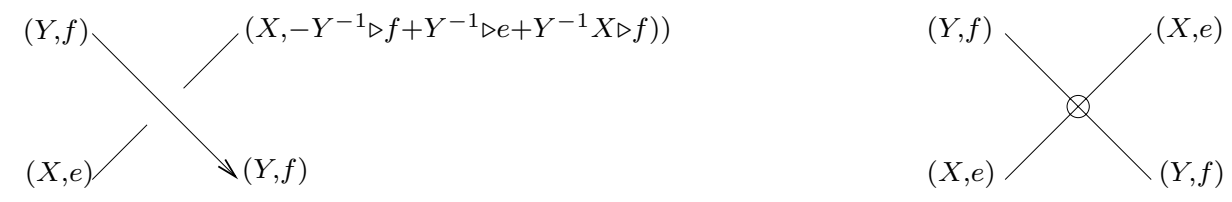

Figure 37. Relations at crossings for the Alexander module Alex $(K)$.
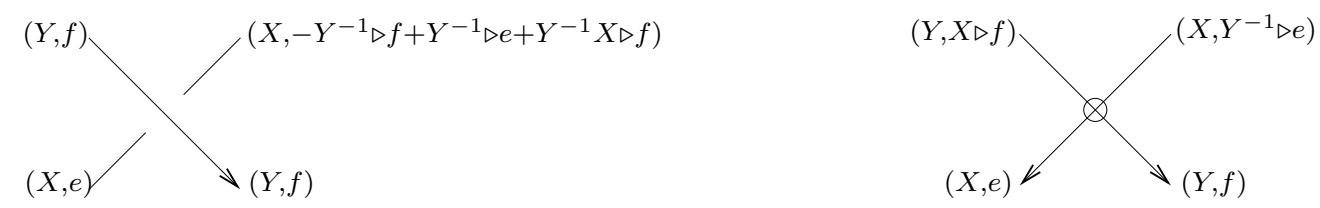

FiguRE 38. Relations at crossings for the module $\operatorname{Alex}^{\prime}(K)$.

3.3.2 Relation with the Alexander module. Let $K$ be a welded virtual link diagram with $n S^{1}$ components, each labelled with an $X_{i} \in\left\{X_{1}, \ldots, X_{n}\right\}$. We can define the Alexander module Alex $(K)$ of $K$, defined as the module over $\kappa_{n}$ with a generator for each connected component of $K$ minus its set of crossings, modulo the relations of Figure 37, obtained from the right handed Wirtinger relations of Figure 5 by applying Fox derivatives; see [BZ03, ch. 9], [Kau87, ch. XI] or [Fox62]. Therefore, if $K$ is a classical one-component knot, then $\operatorname{Alex}(K) \cong \mathbb{Z}\left[X, X^{-1}\right] /\langle\Delta(K)=0\rangle \oplus \mathbb{Z}\left[X, X^{-1}\right]$, where $\Delta(K)$ denotes the Alexander polynomial of $K$; see for example [BZ03, $\S 9 \mathrm{C}]$.

Let $K$ be a welded virtual link diagram. The Alexander module $\operatorname{Alex}(K)$ depends only on the knot group of the welded virtual link defined by $K$, up to isomorphism and reordering of the $S^{1}$-components of $K$.

The module $\operatorname{Alex}(K)$ admits a variant $\operatorname{Alex}^{\prime}(K)$ whose defining relations appear in Figure 38 . Note that the $\kappa_{n}$-module Alex $(K)$ is isomorphic to $\operatorname{Alex}^{\prime}(K)$ whenever $K$ is a classical link diagram.

The module $\operatorname{Alex}^{\prime}(K)$ is invariant under virtual and classical Reidemeister moves. However, $\operatorname{Alex}^{\prime}(K)$ is not invariant under the first forbidden move $F_{1}$; rather it is invariant under the second forbidden move $F_{2}$; see $\S 2.1$.

Given a virtual link diagram $K$, we can define the mirror image $K^{*}$ of it by switching positive to negative crossings, and vice versa, and leaving virtual crossings unchanged. Therefore, the module $\operatorname{Alex}^{\prime}\left(K^{*}\right)$ depends only on the welded virtual knot defined by $K$, up to isomorphism and reordering of the components of $K$.

Theorem 3.4. Let $K$ be a welded virtual link diagram. There exists an isomorphism

$$
\phi: \mathrm{CM}(K) \rightarrow \operatorname{Alex}^{\prime}\left(K^{*}\right) .
$$

Proof. We can suppose that $K$ is the closure of a virtual braid $B$; see [KL06, Kam07]. This avoids needing to deal with the defining relations of $\mathrm{CM}(K)$ at maximal and minimal points. Let $b$ be a connected component of the braid $B$ minus its set of crossings, defining therefore an element $b \in \mathrm{CM}(K)$. The isomorphism $\phi: \operatorname{CM}(K) \rightarrow \operatorname{Alex}^{\prime}\left(K^{*}\right)$ sends $b$ to $Z^{-1} \triangleright b$, where $Z$ is the product of all of the elements $X_{i}$ assigned to the strands of $B$ on the left of $b$ (each belonging to a certain $S^{1}$-component of $K$ ). The remaining details are left to the reader.

The Alexander module of the trefoil knot $3_{1}$ is the module over $Z\left[X, X^{-1}\right]$ with generators $e$ and $f$ and the relation $X^{2} \triangleright(e+f)-X \triangleright(e+f)+(e+f)=0$, thus we have Alex $\left(3_{1}\right)=$ $\mathbb{Z}\left[X, X^{-1}\right] /\left\langle X^{2}-X+1=0\right\rangle \oplus \mathbb{Z}\left[X, X^{-1}\right]$. In particular, it follows $(7)$. 


\section{H. Kauffman and J. FARia Martins}

Let $K$ be a classical one-component knot. By using Theorem 3.4, we can prove that for any automorphic crossed module $\mathcal{G}=(E, G, \triangleright)$, with $G$ abelian, the invariant $\mathcal{H}_{\mathcal{G}}(K)$ is determined by the Alexander module $\operatorname{Alex}(K)$ of $K$, and thus from the Alexander polynomial $\Delta(K)$ of $K$. This is not the case for non-classical links, since the crossed module invariants of the virtual and classical Hopf links $L$ and $H$ (see $\S 3.1$ ) are different, even though they have isomorphic Alexander modules. In fact we have

$$
\operatorname{Alex}(H), \operatorname{CM}(H), \operatorname{Alex}(L)=\frac{\mathbb{Z}\left[X, X^{-1}, Y, Y^{-1}\right] \triangleright e \oplus \mathbb{Z}\left[X, X^{-1}, Y, Y^{-1}\right] \triangleright f}{\langle(X-1) \triangleright f=(Y-1) \triangleright e\rangle},
$$

the module over the ring $Z\left[X, X^{-1}, Y, Y^{-1}\right]$ with two generators $e$ and $f$, and the relation $(X-1) \triangleright$ $f=(Y-1) \triangleright e$, whereas

$$
\mathrm{CM}(L)=\frac{\mathbb{Z}\left[X, X^{-1}, Y, Y^{-1}\right] \triangleright e \oplus \mathbb{Z}\left[X, X^{-1}, Y, Y^{-1}\right] \triangleright f}{\langle Y \triangleright f=f\rangle} .
$$

These last two modules are not isomorphic, as the calculations in $\S 3.1$ certify.

3.3.3 Welded virtual arcs. Let $A$ be a welded virtual arc with a single component. The $\mathbb{Z}\left[X, X^{-1}\right]$-modules $\operatorname{Alex}(A), \operatorname{Alex}^{\prime}\left(A^{*}\right)$ and $\operatorname{CM}(A)$ defined above can still be assigned to $A$, considering the analogue of the relations in Figure 29 at the end-points of $A$, so that the elements of $\operatorname{Alex}(A), \operatorname{Alex}^{\prime}\left(A^{*}\right)$ and $\operatorname{CM}(A)$ assigned to the edges of $A$ incident to its end-points are zero.

Any welded virtual arc $A$ can be obtained as the (incomplete) closure of some braid. Therefore the proof of Theorem 3.4 gives an isomorphism $\phi: \operatorname{CM}(A) \rightarrow \operatorname{Alex}^{\prime}\left(A^{*}\right)$.

Suppose that $A$ is a classical arc sitting in the semiplane $\{z \geqslant 0\}$ of $\mathbb{R}^{3}$, intersecting the plane $\{z=0\}$ at the end-points of $A$, only. Since $A$ is classical we have $\operatorname{Alex}(A)=\operatorname{Alex}^{\prime}(A)$. Let $K$ be the obvious closure of $A$. Then $\operatorname{Alex}(K)=\mathbb{Z}\left[X, X^{-1}\right] /\langle\Delta(K)=0\rangle \oplus \mathbb{Z}\left[X, X^{-1}\right]$, where $\Delta(K)$ is the Alexander polynomial of $K$. Choosing a connected component of $K$ minus its set of crossings, and sending the generator of $\operatorname{Alex}(K)$ it defines to zero, yields a presentation of $\mathbb{Z}\left[X, X^{-1}\right] /\langle\Delta(K)=0\rangle$; see [BZ03, Theorem 9.10]. Comparing with the definition of Alex $(A)$, proves that $\operatorname{Alex}(A)=\mathbb{Z}\left[X, X^{-1}\right] /\langle\Delta(K)=0\rangle$.

Therefore it follows that $\operatorname{CM}(A) \cong Z\left[X, X^{-1}\right] /\langle\Delta(K)=0\rangle$ if $A$ is a classical arc and $K$ is the closure of $A$. The discussion above also implies that if $\mathcal{G}=(E, G, \triangleright)$ is an automorphic crossed module with $G$ abelian then $\mathcal{H}_{\mathcal{G}}(K)=\mathcal{H}_{\mathcal{G}}(A)$ whenever $A$ is a classical one-component arc and $K$ is the one-component knot obtained by closing $A$. This is not the case if $G$ is not abelian.

Problem 2. Let $K$ be a welded virtual link. What is the algebraic topology interpretation of the module $\mathrm{CM}(K)$ in terms of the tube $T(K) \subset S^{4}$ of $K$ ? This should be related to the construction in $[\mathrm{FM} 08]$.

\subsection{Shin Satoh's knot}

In [Sat00], Satoh considered the welded virtual link $S$ displayed in Figure 39. It is a welded virtual knot whose knot group is isomorphic with the knot group of the trefoil knot $3_{1}$. It is possible to prove that $S$ is not equivalent to any classical knot as a welded virtual knot (see [Sat00]); thus Shin Satoh's knot $S$ is not equivalent to the trefoil. See also $\S 3.5 .5$.

Let us calculate the crossed module invariant of Shin Satoh's knot $S$. Let $\mathcal{G}=(E, G, \triangleright)$ be a finite automorphic crossed module. We consider in this case that $G$ is an abelian group, which makes the calculations much easier, since we simply need to calculate the $\mathbb{Z}\left[X, X^{-1}\right]$-module $\operatorname{CM}(S)$. 


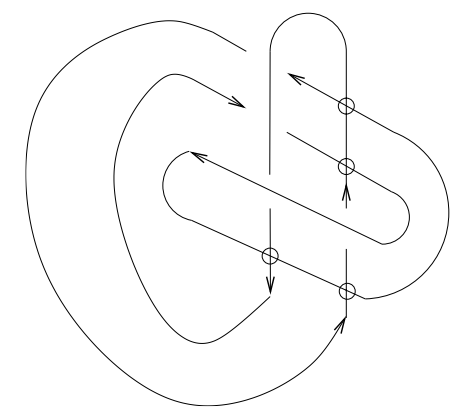

Figure 39. Shin Satoh's knot $S$.

The case when $G$ is non-abelian is considered in $\S 3.5 .5$. Figure 40 permits us to conclude that

$$
\begin{gathered}
\mathcal{H}_{\mathcal{G}}(S)=\#\left\{\begin{array}{l|c}
X \in G ; a, b, c, d \in E \mid \begin{array}{c}
X^{-1} \triangleright(a b) a^{-1} d b^{-1}=c^{-1} \\
X^{-1} \triangleright d^{-1}=b^{-1} \\
X^{-1} \triangleright c^{-1}=a^{-1} \\
X^{-1} \triangleright c c^{-1} X^{-1} \triangleright d X^{-1} \triangleright b^{-1} d^{-1}=X^{-1} \triangleright a
\end{array}
\end{array}\right\} \\
=\#\left\{\begin{array}{l|l}
X \in G ; a, d \in E & \begin{array}{l}
X^{-1} \triangleright a X^{-2} \triangleright d a^{-1} d X^{-1} \triangleright d^{-1}=X \triangleright a^{-1} \\
a X \triangleright a^{-1} X^{-1} \triangleright d X^{-2} \triangleright d^{-1} d^{-1}=X^{-1} \triangleright a
\end{array}
\end{array}\right\} .
\end{gathered}
$$

The two equations in the final expression are equivalent. We obtain, switching to additive notation,

$$
\mathcal{H}_{\mathcal{G}}(S)=\#\left\{X \in G ; a, d \in E \mid X^{-1} \triangleright a-a+X \triangleright a=X^{-1} \triangleright d-d-X^{-2} \triangleright d\right\} .
$$

This should be compared with the crossed module invariant of the trefoil knot $3_{1}$, for $G$ abelian:

$$
\begin{aligned}
\mathcal{H}_{\mathcal{G}}\left(3_{1}\right) & =\#\left\{X \in G ; e, f \in E \mid X^{-3} \triangleright f-X^{-2} \triangleright(e+f)+X^{-1} \triangleright(e+f)=e\right\} \\
& =\#\left\{X \in G ; e, f \in E \mid X^{-3} \triangleright f-X^{-2} \triangleright f+X^{-1} \triangleright f=e-X^{-1} \triangleright e+X^{-2} \triangleright e\right\} \\
& =\#\left\{X \in G ; e, f \in E \mid X^{-2} \triangleright f-X^{-1} \triangleright f+f=X \triangleright e-e+X^{-1} \triangleright e\right\} .
\end{aligned}
$$

Therefore it follows that if $\mathcal{G}=(E, G, \triangleright)$ is an automorphic crossed module with $G$ abelian then

$$
\mathcal{H}_{\mathcal{G}}\left(3_{1}\right)=\mathcal{H}_{\mathcal{G}}(S) \text {. }
$$

We present in the following section (see $\S 3.5 .1$ ) an alternative proof of this fact, which should reassure the reader that the calculations in this paper are correct, despite this being somehow a negative example. We will also see below (see $\S 3.5 .5$ ) that if we take $G$ to be non-abelian, then we can prove that the trefoil knot is not equivalent to Shin Satoh's knot, by using the crossed module invariant.

\subsection{Welded virtual graphs}

3.5.1 Crossed module invariants of knotted surfaces obtained by adding trivial 1-handles. Let $\Sigma \subset S^{4}$ be a knotted surface which we suppose to be connected. The knotted surface $\Sigma^{\prime}$ obtained from $\Sigma$ by adding a trivial 1-handle is defined simply as the connected sum $\Sigma^{\prime}=\Sigma \# T^{2}$, where $T^{2}$ is a torus $S^{1} \times S^{1}$, trivially embedded in $S^{4}$. The non-connected case is totally analogous, but a connected component of $\Sigma$ must be chosen. A movie of $\Sigma^{\prime}$ is obtained from a movie of $\Sigma$ by choosing a strand of the movie of $\Sigma$ belonging to the chosen component of $\Sigma$, and making the modification shown in Figure 41. The straightforward proof of the following theorem is left to the reader. 
L. H. Kauffman And J. FARia Martins

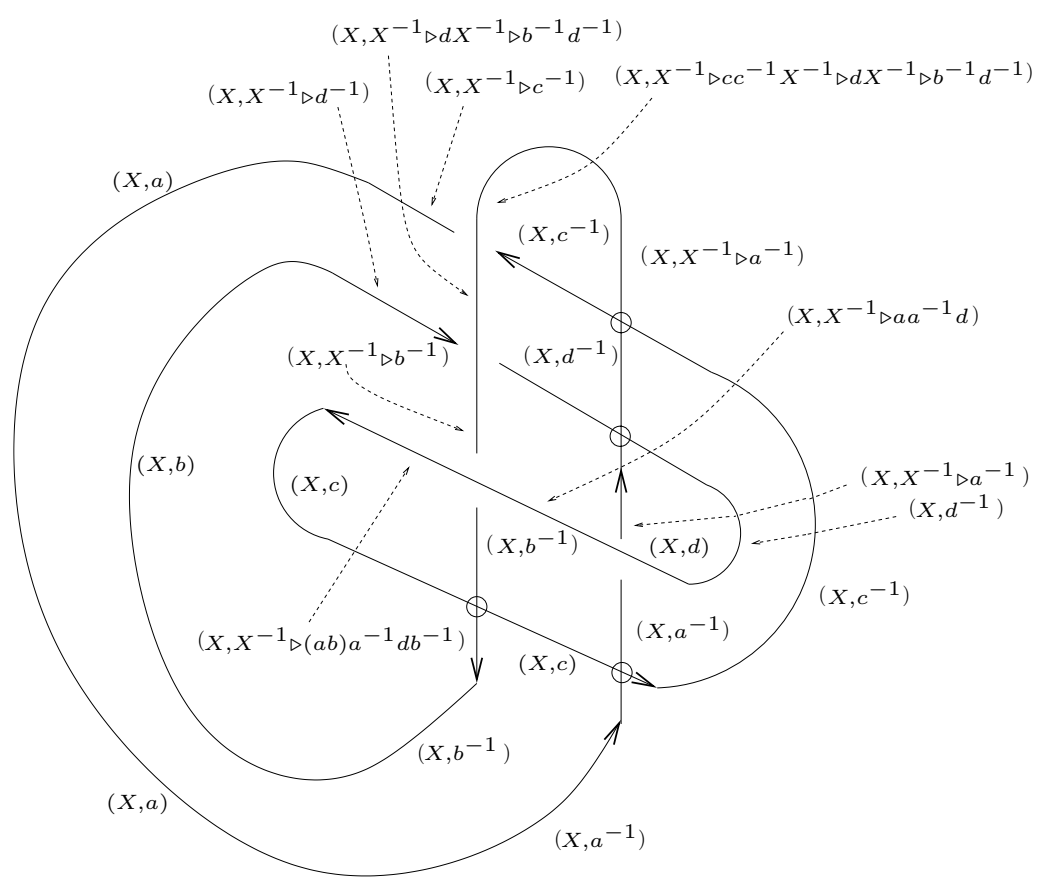

Figure 40. Calculation of the crossed module invariant of Shin Satoh's knot $S$ for $G$ abelian.
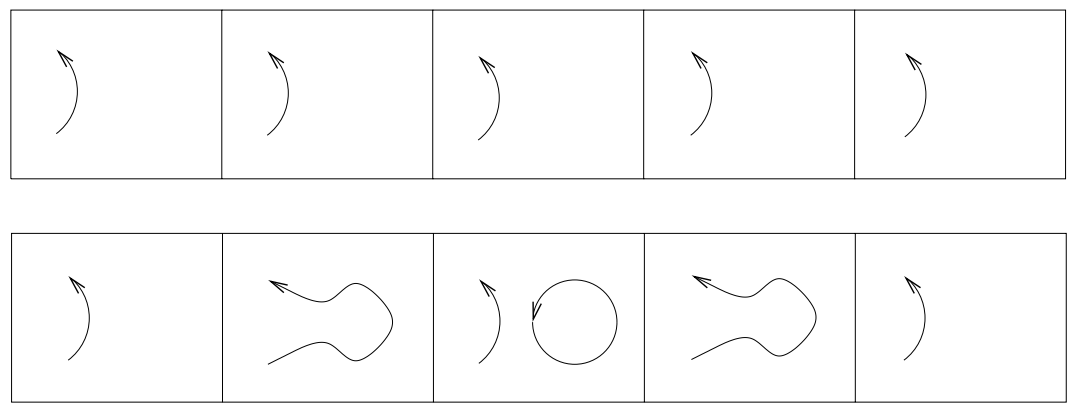

Figure 41. Adding a trivial 1-handle to a knotted surface. On the top we display the original movie, and on the bottom the new movie, both read from left to right. A concise description of this modification is fission saddle, fusion saddle.

Theorem 3.5. Let $\mathcal{G}=(E \stackrel{\partial}{\rightarrow} G, \triangleright)$ be a finite crossed module. If the oriented knotted surface $\Sigma^{\prime}$ is obtained from the oriented knotted surface $\Sigma$ by adding a trivial 1-handle then

$$
I_{\mathcal{G}}\left(\Sigma^{\prime}\right)=\frac{(\# \operatorname{ker} \partial)^{2}}{(\# E)^{2}} I_{\mathcal{G}}(\Sigma)
$$

thus in particular $I_{\mathcal{G}}(\Sigma)=I_{\mathcal{G}}\left(\Sigma^{\prime}\right)$ whenever $\mathcal{G}$ is automorphic.

The tube $T(S)$ of Shin Satoh's knot $S$ is obtained from the spun trefoil (the tube $T\left(3_{1}^{\prime}\right)$ of the trefoil arc $3_{1}^{\prime}$ ) by adding a trivial 1-handle; see [Sat00] or $\S 3.5 .2$. This fact together with (10) proves that $\mathcal{H}_{\mathcal{G}}\left(3_{1}\right)=\mathcal{H}_{\mathcal{G}}(S)$, whenever $\mathcal{G}=(E, G, \triangleright)$ is a finite automorphic crossed module with $G$ abelian, as already proved by other means; see $\S 3.4$. Here $3_{1}$ is the trefoil knot. 


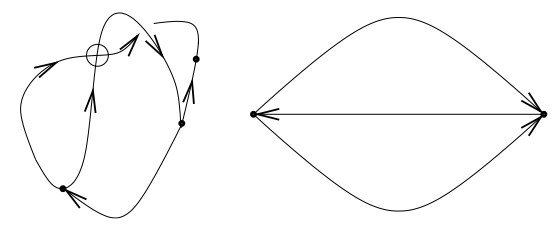

Figure 42. A welded virtual graph.

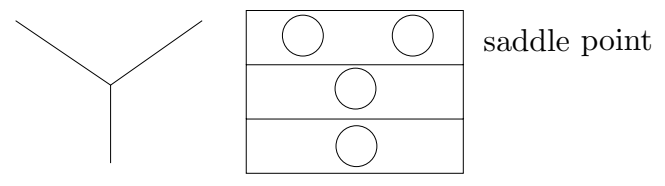

FiguRE 43. The tube of a virtual graph at a 3 -valent vertex (movie version). As usual, all circles are oriented counterclockwise.

FiguRE 44. The tube of a virtual graph at a 3-valent vertex; broken surface diagram version of the movie of Figure 43.

3.5.2 Definition of welded virtual graphs. Let $K$ be an oriented virtual graph diagram. Note that $K$ may have some bivalent vertices where the orientation of an edge of $K$ may change; however, there cannot be a change of orientation of a strand at a crossing; see Figure 42.

Given a virtual graph diagram $K$, we can define the tube $T(K)$ of it exactly in the same way as the tube of a virtual link or arc is defined. We consider the type of movie of Figure 43 at the 3-valent vertices. For the broken surface diagram version of this, see Figure 44. We proceed analogously for $n$-valent vertices if $n>3$. The 2 -valent vertices do not affect the calculation of $T(K)$. On the other hand 1 -valent vertices were already considered in the case of virtual arcs.

It is easy to see that the tube $T(K)$ of a virtual graph is invariant under the moves defining welded virtual knots and arcs; see $\S \S 2.1$ and 2.2.1. In addition, $T(K)$ is invariant under the moves shown in Figure 45. Note that if a strand in Figure 45 is drawn without orientation, then this means that the corresponding identity is valid for any choice of orientation.

The invariance under the first, second and fifth moves is immediate. The invariance under the third and fourth moves follows from Figures 6 and 44, by sliding the cylinder that goes inside the other cylinder towards the end strand, in the obvious way, as shown in Figure 46. It is strictly necessary that the edges incident to the vertex under consideration have compatible orientations in the sense shown in Figure 45. Note that otherwise the crossing information in the corresponding initial and final broken surface diagrams in Figure 46 would not be compatible.

The invariance of $T(K)$ under the penultimate moves of Figure 45 follows from the same argument that proves invariance under the classical and virtual Reidemeister-I moves.

Definition 3.6 (Welded virtual graph). The moves on oriented virtual graph diagrams of Figure 45, together with the ones defining welded virtual knots and welded virtual arcs, define what we called a welded virtual graph. 
L. H. Kauffman and J. Faria Martins
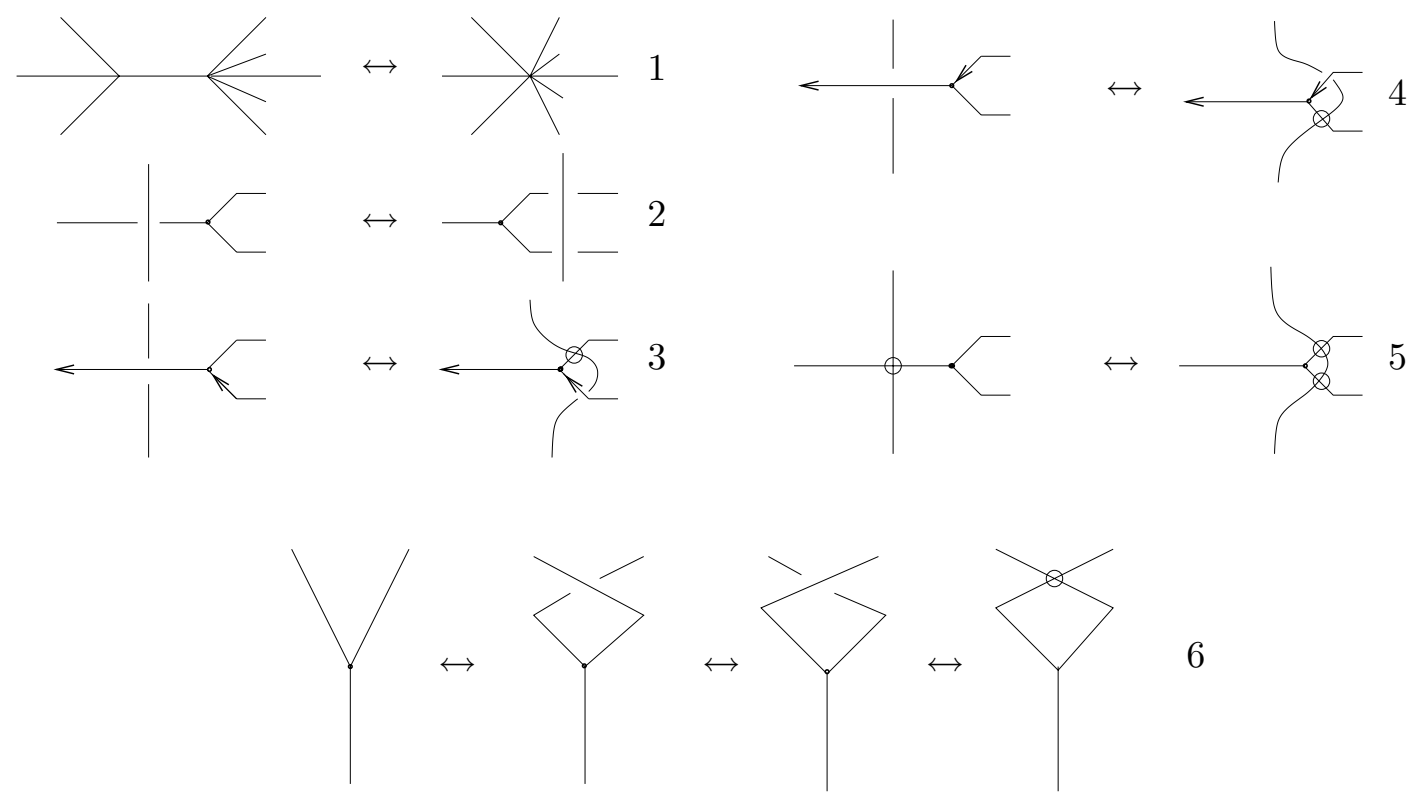

6

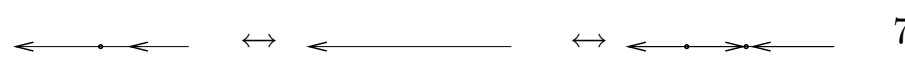

Figure 45. Moves defining welded virtual graphs. Notice that the third and fourth moves have a variant for which the direction of each strand is reversed. However, these moves are a consequence of the remaining.

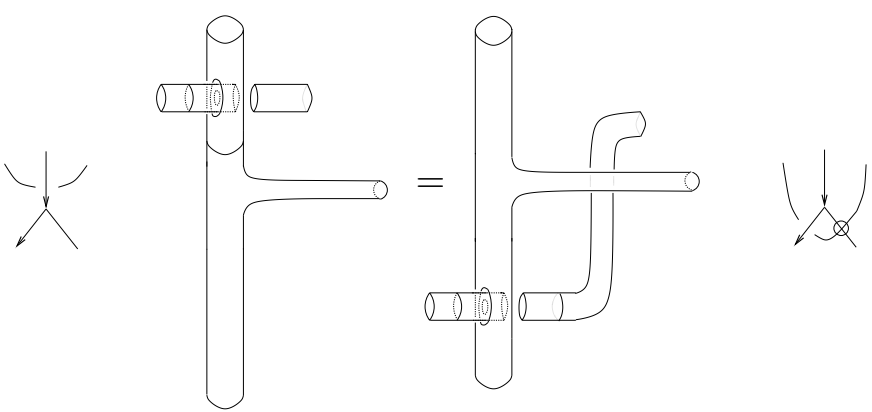

FiguRE 46. An identity between broken surface diagrams of knotted surfaces (reverse orientation of the fourth move of Figure 45).
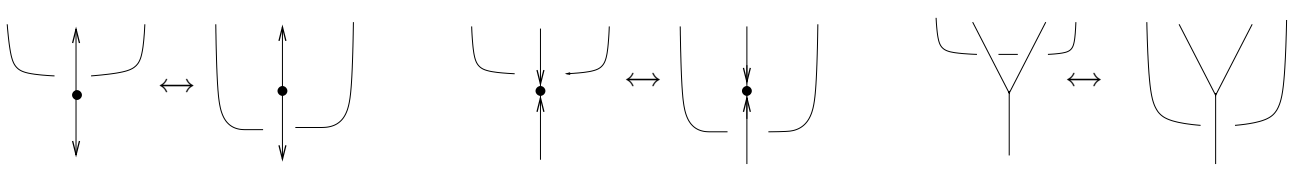

FiguRE 47. Forbidden moves.

Note that the moves of Figure 47 are not allowed.

If $K$ is a welded virtual graph, then a welded virtual graph $K^{\prime}$ for which the tube $T\left(K^{\prime}\right)$ of $K^{\prime}$ is obtained from $T(K)$ by adding a trivial 1-handle is obtained from $K$ by choosing a string of $K$ (in the correct component) and doing the transition shown in Figure 48 (adding a trivial 1-handle to a welded virtual graph). 


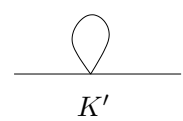

FiguRE 48. Adding a trivial 1-handle to a welded virtual graph. On the left we display the original graph.
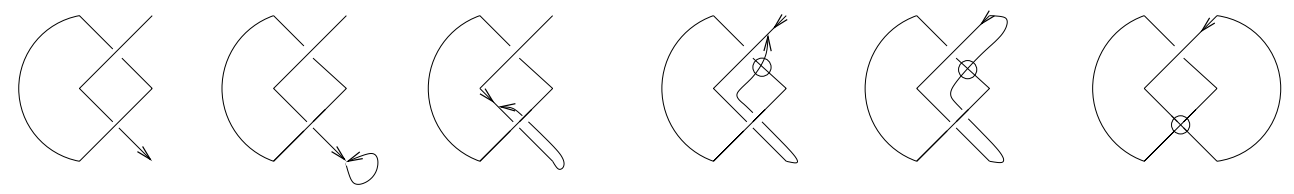

Figure 49. Adding a trivial 1-handle to the Hopf arc yields the virtual Hopf link.

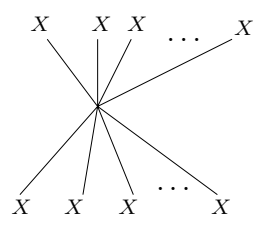

FiguRE 50. The relations satisfied by the knot group of a welded virtual graph at a vertex.

For example, consider the Hopf arc $H A$ defined in Exercise 2. Then adding a trivial 1-handle to the unclosed component of it yields the virtual Hopf link $L$; see Figure 49. Note the usage of the moves of Figure 45.

Let $G_{1}$ be a welded virtual graph such that, topologically, $G_{1}$ is the union of circles $S^{1}$ and intervals $I=[0,1]$. Suppose that $G_{1}^{\prime}$ is obtained from $G_{1}$ by adding a trivial 1-handle to an $I$-component of it. Then we can always use the moves of Figure 45 to find a graph $G_{2}$, equivalent to $G_{1}^{\prime}$ as a welded virtual graph, but so that, topologically, $G_{2}$ is the union of circles $S^{1}$ and intervals $I$. This was exemplified above for the case of the Hopf arc $H A$, and should be compared with the method indicated in [Sat00, p. 541].

It is a good exercise to verify that adding a trivial 1-handle to the trefoil arc yields Shin Satoh's knot.

3.5.3 The fundamental group of the complement. The (combinatorial) fundamental group of a welded virtual graph complement (the knot group) is defined in the same way as the knot group of a virtual knot or arc. However, we consider the relations of Figure 50 at the vertices of a graph (the edges incident to a vertex may carry any orientation). Note that this is in sharp contrast with the classical fundamental group of graph complements. In fact, we can easily find examples of welded virtual graphs for which the classical and virtual knot groups are different. The $\theta$-graph which appears in Figure 42 is such an example.

It is not difficult to see that the knot group is an invariant of welded virtual graphs. Moreover the tube map $K \mapsto T(K)$ preserves knot groups.

Suppose that the graph $K^{\prime}$ is obtained from $K$ by adding a trivial 1-handle. We can see that the knot groups of $K$ and $K^{\prime}$ are isomorphic, thus also that the fundamental groups of the complements of the tubes $T(K)$ and $T\left(K^{\prime}\right)$ in $S^{4}$ are isomorphic. This can easily be proved directly.

Given an arc $A$ embedded in the upper semiplane $\{z \geqslant 0\}$ of $\mathbb{R}^{3}$, intersecting the plane $\{z=0\}$ at the end-points of $A$, only, there exist two knotted tori naturally associated to $A$. The first one is 
L. H. Kauffman And J. FARia Martins

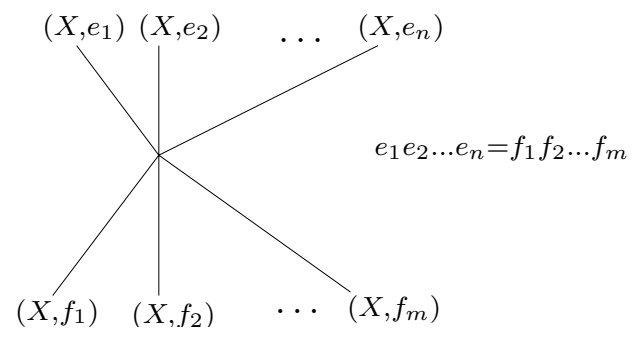

FiguRE 51. Reduced $\mathcal{G}$-colourings of a welded virtual graph diagram at a vertex.

obtained from the tube $T(A)$ of $A$ by adding a trivial 1-handle, and a virtual knot $c_{1}(A)$ representing it can be easily determined from $A$ using the method indicated in [Sat00] and $\S$ 3.5.2. In the second one, one simply closes $A$ in the obvious way, obtaining $c_{2}(A)$, before taking the tube of it. If $A$ is a classical arc, with only one component, then we have that the fundamental groups of the complements of the knotted surfaces $T(A), T\left(c_{1}(A)\right)$ and $T\left(c_{2}(A)\right)$ are all isomorphic. This also happens if we allow $A$ to have more than one component, as long as all the other components are diffeomorphic to $S^{1}$. However, it is necessary that $A$ be classical.

The pairs of welded virtual knots $\left(c_{1}(A), c_{2}(A)\right)$, one for each classical one-component arc $A$, provide a family of welded virtual knots with the same knot group. For example if $3_{1}^{\prime}$ is the trefoil arc, then $c_{2}\left(3_{1}^{\prime}\right)$ is the trefoil knot $3_{1}$, whereas $c_{1}\left(3_{1}^{\prime}\right)$ is Shin Satoh's knot. These two can be proven to be non-equivalent by using the crossed module invariant; see $\S 3.5 .5$. See also $\S \S 3.6$ to 3.10 for other analogous examples.

Problem 3. Under which circumstances are the welded virtual knots $c_{1}(A)$ and $c_{2}(A)$ equivalent? What can one say about their tubes in $S^{4}$.

3.5.4 Crossed module invariants of welded virtual graphs. Let $\mathcal{G}=(E, G, \triangleright)$ be an automorphic finite crossed module. The invariant $\mathcal{H}_{\mathcal{G}}$ of welded virtual knots, or arcs, extends in a natural way to an invariant of welded virtual graphs $K$, by considering

$$
\mathcal{H}_{\mathcal{G}}(K) \doteq I_{\mathcal{G}}(T(K)),
$$

where $I_{\mathcal{G}}$ is the four-dimensional invariant defined in $\S 2.3$. As before, $\mathcal{H}_{\mathcal{G}}(K)$ can be calculated directly from a diagram of $K$.

Definition 3.7. Let $\mathcal{G}=(E, G, \triangleright)$ be a finite automorphic crossed module. Let $K$ be an oriented welded virtual graph diagram chosen so that the projection on the second variable is a Morse function in $K$. A reduced $\mathcal{G}$-colouring of $K$ is given by an assignment of a pair $(X, e) \in G \times E$ to each arc of $G$ minus its set of critical points, crossings and vertices, satisfying the conditions already shown for virtual knot and arc diagrams, and the relation displayed in Figure 51.

We have the following result.

Theorem 3.8. Let $\mathcal{G}=(E, G, \triangleright)$ be a finite automorphic crossed module. Let also $K$ be an oriented welded virtual graph diagram. The quantity

$$
\begin{aligned}
\mathcal{H}_{\mathcal{G}}(K)= & \#\{\text { reduced } \mathcal{G} \text {-colourings of } K\} \# E^{\#\{\text { caps }\}} \# E^{-\#\{\text { cups }\}} \\
& \# E^{\#\{\text { pointing upward 1-valent vertices of } K\}} \\
& \prod_{n \text {-valent }} \# E^{1-\# \text { vertices } v \text { of } K} n
\end{aligned}
$$

coincides with $I_{\mathcal{G}}(T(K))$, and therefore defines an invariant of welded virtual graphs. 


\section{INVARIANTS OF WELDED VIRTUAL KNOTS}

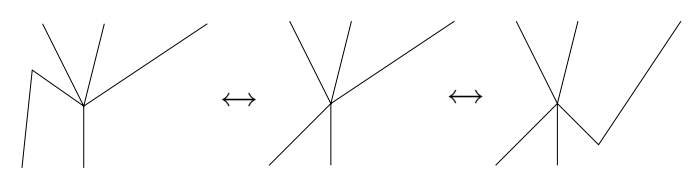

FIGURE 52. One type of Yetter's moves capturing planar isotopy of graph diagrams.

TABLE 1 . The crossed module invariant of the welded virtual knots $3_{1}$ and $S$.

\begin{tabular}{cccccc}
\hline Knot & $\mathcal{H}_{\mathcal{G}_{2,2}}$ & $\mathcal{H}_{\mathcal{G}_{2,3}}$ & $\mathcal{H}_{\mathcal{G}_{2,4}}$ & $\mathcal{H}_{\mathcal{G}_{2,5}}$ & $\mathcal{H}_{\mathcal{G}_{2,7}}$ \\
\hline $3_{1}$ & 96 & 4320 & 24576 & 132000 & 2272032 \\
$S$ & 96 & 4752 & 27648 & 168000 & 2765952 \\
\hline
\end{tabular}

Exercise 4. Check directly that $\mathcal{H}_{\mathcal{G}}$ is a topological invariant of welded virtual graphs. Together with the moves of Figure 45, as well as the moves defining welded virtual knots and arcs, one still needs to check invariance under planar isotopy. Planar isotopies of graph diagrams are captured by Yetter's moves shown in Figure 28, as well as Figure 52; see [Yet89] and [FY89].

Exercise 5. Check directly that $\mathcal{H}_{\mathcal{G}}$ is invariant under addition of trivial 1-handles, as shown in Figure 48; cf. Theorem 3.5.

3.5.5 The trefoil knot is not equivalent to Shin Satoh's knot. We now use the extension of the crossed module invariant to welded virtual graphs to prove that Shin Satoh's knot $S$ is not equivalent to the trefoil knot $3_{1}$. Let $3_{1}^{\prime}$ be the trefoil arc. Recall that $S$ is obtained from $3_{1}^{\prime}$ by adding a trivial 1-handle, in other words $S=c_{1}\left(3_{1}^{\prime}\right)$; see $\S 3.5 .3$. Therefore, whenever $\mathcal{G}=(E, G, \triangleright)$ is a finite automorphic crossed module we have

$$
\mathcal{H}_{\mathcal{G}}\left(3_{1}^{\prime}\right)=\mathcal{H}_{\mathcal{G}}(S) .
$$

In particular, from (8)

$$
\mathcal{H}_{\mathcal{G}}(S)=\mathcal{H}_{\mathcal{G}}\left(3_{1}^{\prime}\right)=\# E \#\left\{\begin{array}{l|l}
X, Y \in G ; e \in E & \begin{array}{c}
X Y X=Y X Y \\
-X Y \triangleright e+Y \triangleright e=e
\end{array}
\end{array}\right\}
$$

note that we switched to additive notation. Also, from (6):

$$
\mathcal{H}_{\mathcal{G}}\left(3_{1}\right)=\#\left\{\begin{array}{c|c}
X, Y \in G ; e, f \in E & \begin{array}{c}
X Y X=Y X Y \\
Y X Y \triangleright f-X Y \triangleright(e+f)+Y \triangleright(e+f)=e
\end{array}
\end{array}\right\} .
$$

A natural example of a finite automorphic crossed module $\mathcal{G}=(E, G, \triangleright)$ with $G$ non-abelian is constructed by taking $G=\mathrm{GL}_{n}\left(\mathbb{Z}_{p}\right)$ and $E=\left(\mathbb{Z}_{p}\right)^{n}$. Here $\mathrm{GL}_{n}\left(\mathbb{Z}_{p}\right)$ denotes the group of $n \times n$ matrices in $\mathbb{Z}_{p}$ with invertible determinant, where $p$ is a positive integer. The action of $\mathrm{GL}_{n}\left(\mathbb{Z}_{p}\right)$ in $\left(\mathbb{Z}_{p}\right)^{n}$ is taken to be the obvious one. Denote these crossed modules by $\mathcal{G}(n, p)$.

Computations with Mathematica prove that $\mathcal{H}_{\mathcal{G}(n, p)}\left(3_{1}\right) \neq \mathcal{H}_{\mathcal{G}(n, p)}(S)$, for example for $p=$ $3,4,5,7$ and $n=2$; see Table 1 . This proves that the crossed module invariant distinguishes the trefoil knot from Shin Satoh's knot, even though they have the same knot group.

\subsection{Figure of eight knot}

Let $\mathcal{G}=(E, G, \triangleright)$ be a finite automorphic crossed module. Let us calculate the crossed module invariant $\mathcal{H}_{\mathcal{G}}\left(4_{1}\right)$ of the figure of eight knot $4_{1}$. This calculation appears in Figure 53. This permits 


\section{H. KaufFman And J. FARia Martins}

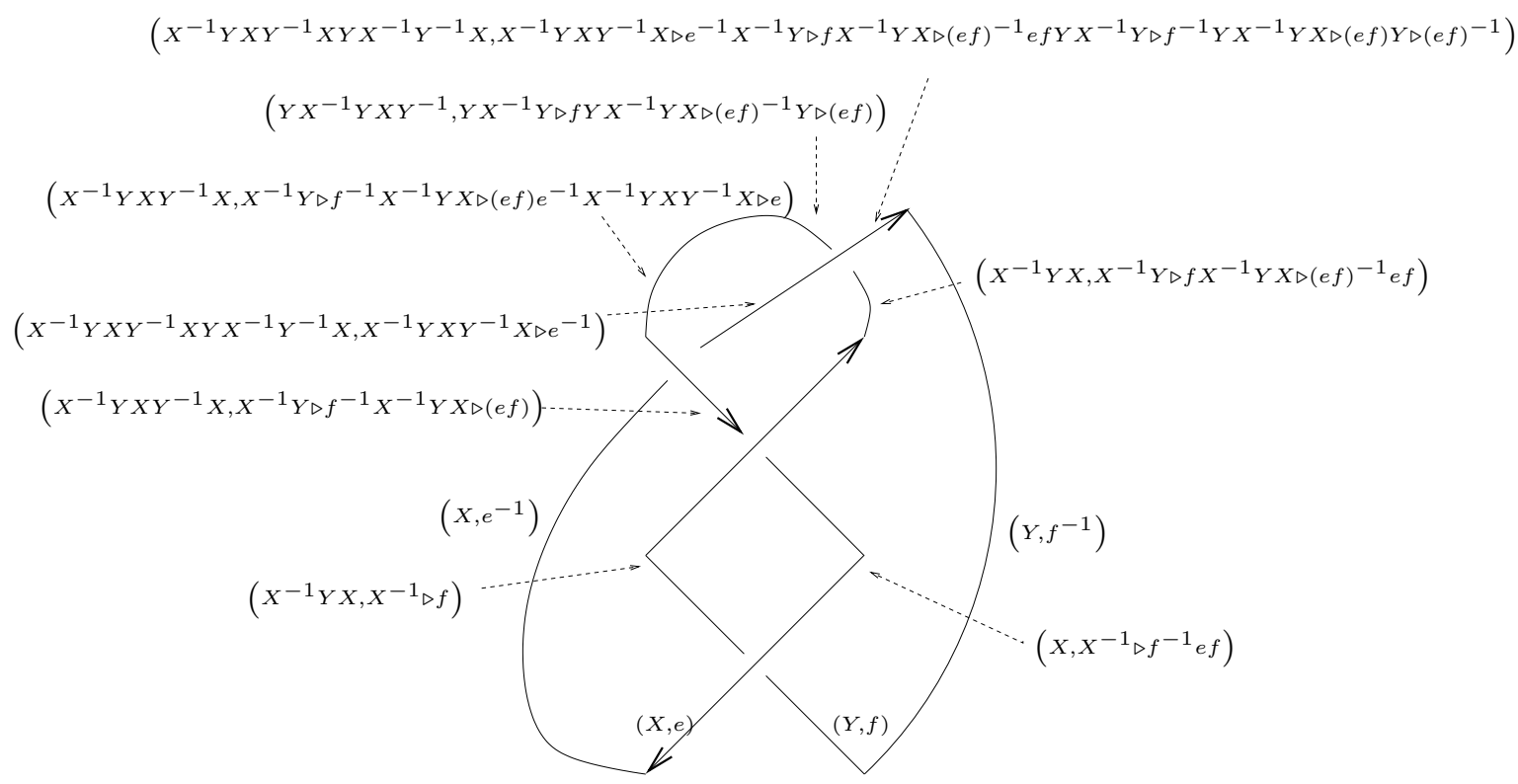

FiguRE 53. Calculation of the crossed module invariant of the figure of eight knot $4_{1}$.

us to conclude that, if $\mathcal{G}=(E, G, \triangleright)$ is an automorphic crossed module, then

$$
\mathcal{H}_{\mathcal{G}}\left(4_{1}\right)=\#\left\{\begin{array}{c|c}
X, Y \in G ; e, f \in E & \begin{array}{c}
X^{-1} Y X Y^{-1} X Y X^{-1} Y^{-1} X=Y \\
X^{-1} Y X Y^{-1} X \triangleright e^{-1} X^{-1} Y \triangleright f X^{-1} Y X \triangleright(e f)^{-1} e f Y X^{-1} Y \triangleright f^{-1} Y X^{-1} Y X \triangleright(e f) Y \triangleright(e f)^{-1}=f \\
X^{-1} Y X Y^{-1} X=Y X^{-1} Y X Y^{-1} \\
X^{-1} Y \triangleright f^{-1} X^{-1} Y X \triangleright(e f) e^{-1} X^{-1} Y X Y^{-1} X \triangleright e=Y X^{-1} Y \triangleright f^{-1} Y X^{-1} Y X \triangleright(e f) Y \triangleright(e f)^{-1}
\end{array}
\end{array} .\right.
$$

Note that the first pair of equations which appear in the previous formula is equivalent to the second one. In the case when $G$ is abelian, the previous formula simplifies to (passing to additive notation)

$$
\begin{aligned}
\mathcal{H}_{\mathcal{G}}\left(4_{1}\right) & =\#\left\{X \in G ; e, f \in E \mid\left(X^{2}-3 X+1\right) \triangleright e=\left(-X^{2}+3 X-1\right) \triangleright f\right\} \\
& =\# E \#\left\{X \in G ; e \in E \mid\left(X^{2}-3 X+1\right) \triangleright e=0\right\},
\end{aligned}
$$

as it should, since the Alexander polynomial of the figure of eight knot is $\Delta\left(4_{1}\right)=X^{2}-3 X+1$; see $\S 3.3 .2$.

The value of the crossed module invariant for the figure of eight arc $4_{1}^{\prime}$ (Figure 54), for $G$ not necessarily abelian, can be obtained from (16) by making $f=1$, and inserting the relevant normalisation factors. This yields

$\mathcal{H}_{\mathcal{G}}\left(4_{1}^{\prime}\right)=\# E \#\left\{X, Y \in G ; e \in E \mid \begin{array}{c}X^{-1} Y X Y^{-1} X Y X^{-1} Y^{-1} X=Y \\ X^{-1} Y X Y^{-1} X \triangleright e^{-1} X^{-1} Y X \triangleright e^{-1} e Y X^{-1} Y X \triangleright e Y \triangleright e^{-1}=1\end{array}\right\}$.

Consider the welded virtual knot $c_{1}\left(4_{1}^{\prime}\right)$ obtained from the figure of eight arc $4_{1}^{\prime}$ by adding a trivial 1-handle to it; see $\S 3.5 .2$. This welded virtual knot appears in Figure 54. By using Theorem 3.5, it thus follows that for any finite automorphic crossed module $\mathcal{G}$ we have $\mathcal{H}_{\mathcal{G}}\left(c_{1}\left(4_{1}^{\prime}\right)\right)=\mathcal{H}_{\mathcal{G}}\left(4_{1}^{\prime}\right)$; see also $\S 3.5 .4$. Recall that by the discussion in $\S 3.5 .3$, the knot groups of the welded virtual knots $4_{1}=c_{2}\left(4_{1}^{\prime}\right)$ and $c_{1}\left(4_{1}^{\prime}\right)$ are isomorphic.

Consider the crossed modules $\mathcal{G}_{(n, p)}$, where $p$ and $n$ are positive integers, obtained from $\operatorname{GL}_{n}\left(\mathbb{Z}_{p}\right)$ acting on $\left(\mathbb{Z}_{p}\right)^{n}$, defined in $\S 3.5 .5$. Computations with Mathematica prove that $\mathcal{H}_{\mathcal{G}_{(n, p)}}\left(c_{1}\left(4_{1}^{\prime}\right)\right) \neq$ $\mathcal{H}_{\mathcal{G}_{(n, p)}}\left(4_{1}\right)$ for $p=3$ or $p=7$; see Table 2 . This proves that the welded virtual knots $4_{1}=c_{2}\left(4_{1}^{\prime}\right)$ and $c_{1}\left(4_{1}^{\prime}\right)$ are not equivalent, even though they have the same knot groups. 

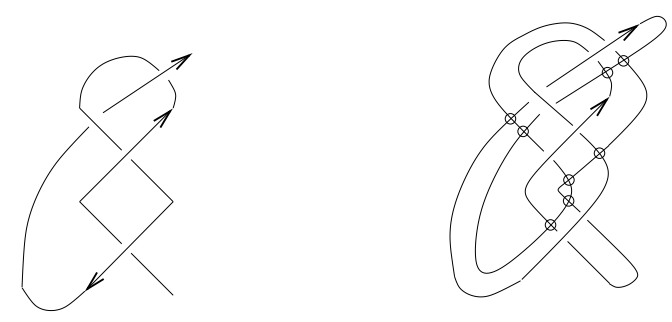

FigurE 54 . The figure of eight arc $4_{1}^{\prime}$ and the welded virtual knot $c_{1}\left(4_{1}^{\prime}\right)$ obtained from it by adding a trivial 1-handle.

TABLE 2. The crossed module invariant of the welded virtual knots $4_{1}$ and $c_{1}\left(4_{1}^{\prime}\right)$.

\begin{tabular}{cccccc}
\hline Knot & $\mathcal{H}_{\mathcal{G}_{2,2}}$ & $\mathcal{H}_{\mathcal{G}_{2,3}}$ & $\mathcal{H}_{\mathcal{G}_{2,4}}$ & $\mathcal{H}_{\mathcal{G}_{2,5}}$ & $\mathcal{H}_{\mathcal{G}_{2,7}}$ \\
\hline $4_{1}$ & 48 & 3024 & 15360 & 228000 & 1876896 \\
$c_{1}\left(4_{1}^{\prime}\right)$ & 48 & 3456 & 15360 & 228000 & 2272032 \\
\hline
\end{tabular}

TABLE 3 . The crossed module invariant of the welded virtual knots $5_{1}$ and $c_{1}\left(5_{1}^{\prime}\right)$.

\begin{tabular}{cccccc}
\hline Knot & $\mathcal{H}_{\mathcal{G}_{2,2}}$ & $\mathcal{H}_{\mathcal{G}_{2,3}}$ & $\mathcal{H}_{\mathcal{G}_{2,4}}$ & $\mathcal{H}_{\mathcal{G}_{2,5}}$ & $\mathcal{H}_{\mathcal{G}_{2,7}}$ \\
\hline $5_{1}$ & 24 & 432 & 1536 & 168000 & 98784 \\
$c_{1}\left(5_{1}^{\prime}\right)$ & 24 & 432 & 1536 & 204000 & 98784 \\
\hline
\end{tabular}

\subsection{The Solomon seal knot}

Let $\mathcal{G}=(E, G, \triangleright)$ be an automorphic finite crossed module. The crossed module invariant of the $(5,2)$-torus knot $55_{1}$ (the Solomon seal knot) is calculated in Figure 55. This permits us to conclude that

$$
\begin{aligned}
& \mathcal{H}_{\mathcal{G}}\left(5_{1}\right) \\
& =\#\left\{X, Y \in G ; e, f \in E \mid \begin{array}{c}
X Y X Y X=Y X Y X Y \\
Y X Y X Y \triangleright f X Y X Y \triangleright(e f)^{-1} Y X Y \triangleright(e f) X Y \triangleright(e f)^{-1} Y \triangleright(e f)=e
\end{array}\right\} .
\end{aligned}
$$

Note that if the crossed module $\mathcal{G}=(E, G, \triangleright)$ is such that $G$ is abelian, then the previous expression simplifies to

$$
\mathcal{H}_{\mathcal{G}}\left(5_{1}\right)=\# E \#\left\{X \in G ; e \in E \mid X^{4} \triangleright e-X^{3} \triangleright e+X^{2} \triangleright e-X \triangleright e+e=0\right\},
$$

as it should, since the Alexander polynomial of the knot $5_{1}$ is $\Delta\left(5_{1}\right)=X^{4}-X^{3}+X^{2}-X+1$.

The crossed module invariant of the Solomon seal arc $5_{1}^{\prime}$, and the welded virtual knot $c_{1}\left(5_{1}^{\prime}\right)$ obtained from it by adding a trivial 1-handle, each presented in Figure 56, can be obtained from this calculation by making $f=1$, and inserting the remaining normalisation factors. Therefore it follows that

$$
\mathcal{H}_{\mathcal{G}}\left(5_{1}^{\prime}\right)=\# E \#\left\{\begin{array}{l|c}
X, Y \in G ; e \in E & \begin{array}{c}
X Y X X=Y X Y X Y \\
X Y X Y \triangleright e^{-1} Y X Y \triangleright e X Y \triangleright e^{-1} Y \triangleright e=e
\end{array}
\end{array}\right\} .
$$

Computations with Mathematica show that $\mathcal{H}_{\mathcal{G}_{(n, p)}}\left(c_{1}\left(5_{1}^{\prime}\right)\right) \neq \mathcal{H}_{\mathcal{G}_{(n, p)}}\left(5_{1}\right)$ for $n=2$ and $p=5$; see Table 3. Therefore the pair $\left(5_{1}, c_{1}\left(5_{1}\right)\right)$ is a pair of welded virtual knots with the same knot group, but distinguished by their crossed module invariant. 


\section{H. KaufFman And J. FARia Martins}

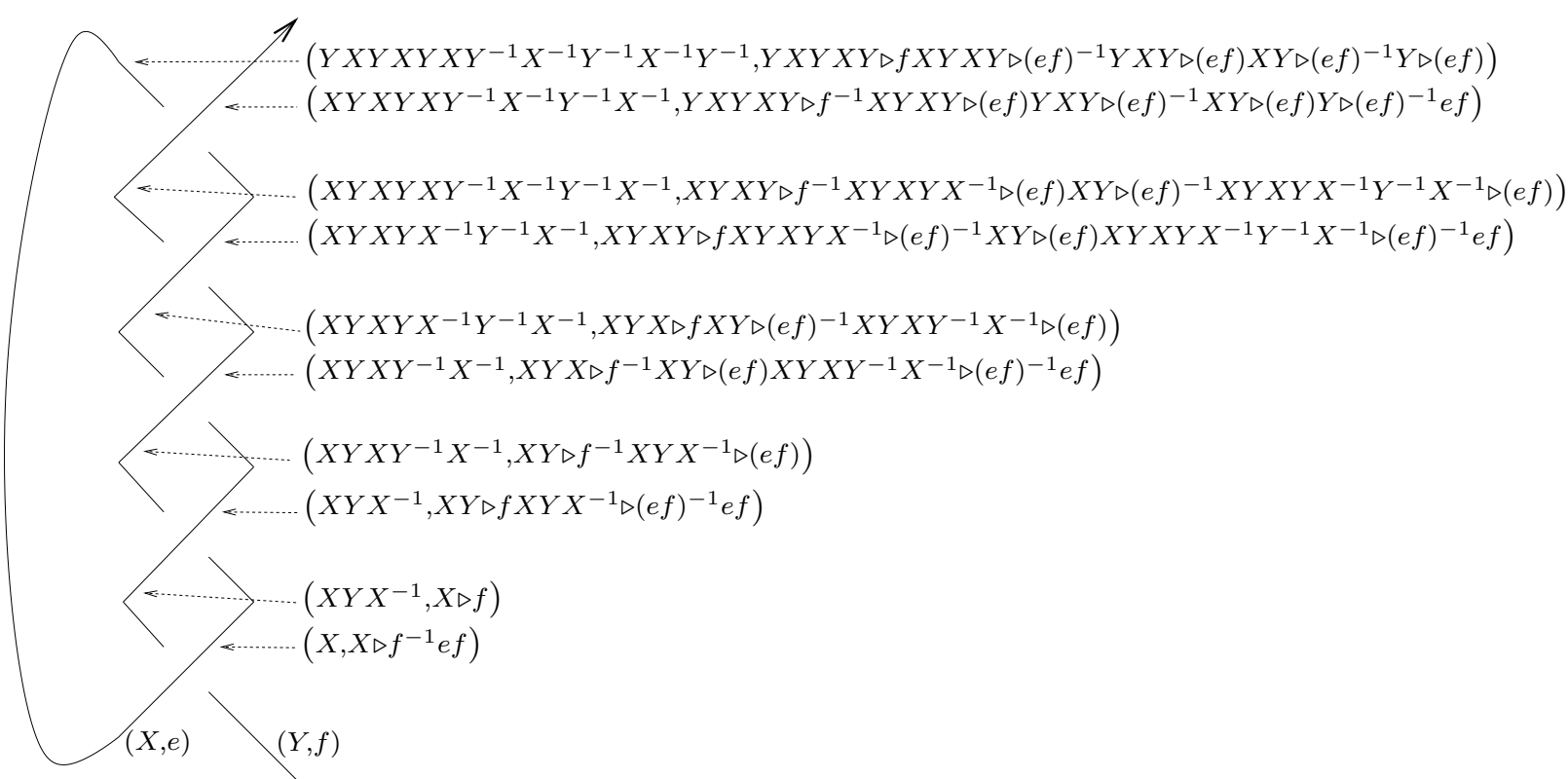

Figure 55. Calculation of the crossed module invariant of the torus knot $55_{1}$. In the top two colourings, we are using the fact that $X Y X Y X=Y X Y X Y$.
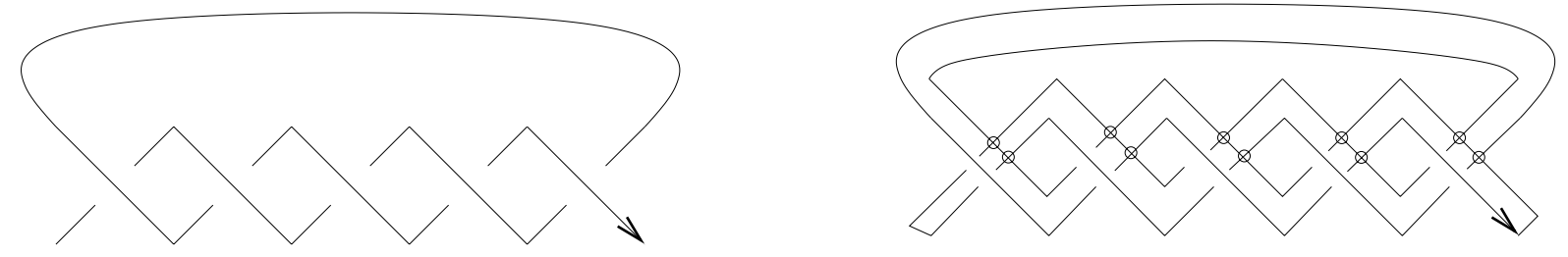

FiguRE 56 . The Solomon seal arc $5_{1}^{\prime}$ and the welded virtual knot $c_{1}\left(5_{1}^{\prime}\right)$ obtained by adding a trivial 1-handle to it.

\subsection{The 2-bridge knot $5_{2}$ (stevedore)}

We now consider the 2-bridge knot $5_{2}$ and the 2-bridge arc $5_{2}^{\prime}$, depicted in Figure 57. Let us calculate their crossed module invariant. Suppose that $\mathcal{G}=(E, G, \triangleright)$ is a finite automorphic crossed module. The calculation of $\mathcal{H}_{\mathcal{G}}\left(5_{2}\right)$ appears in Figure 58. From this it follows that

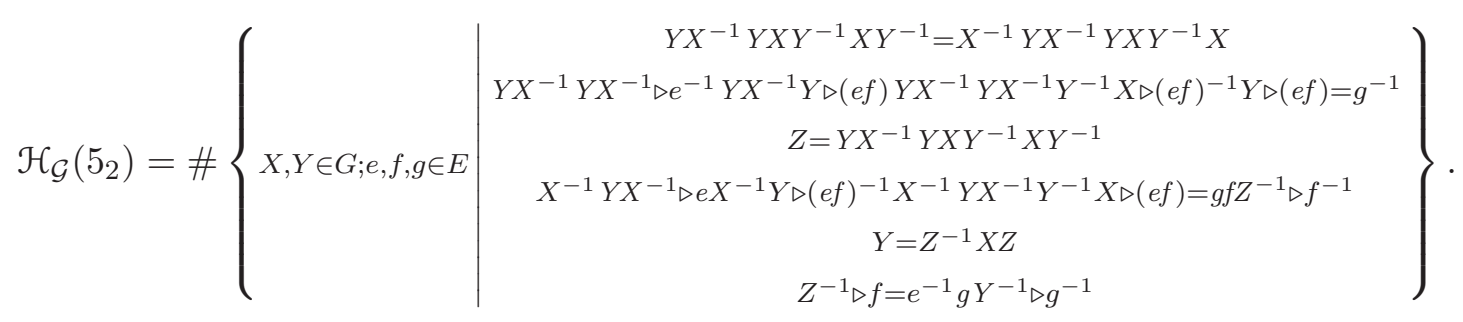

Note that the last two equations in the previous formula follow from the remaining. When $G$ is abelian, the previous expression reduces to

$$
\mathcal{H}_{\mathcal{G}}\left(5_{2}\right)=\# E \#\left\{X \in G ; e \in E \mid 2 X^{2} \triangleright e-3 e+2 X^{-1} \triangleright e=0\right\},
$$




\section{INVARIANTS OF WELDED VIRTUAL KNOTS}
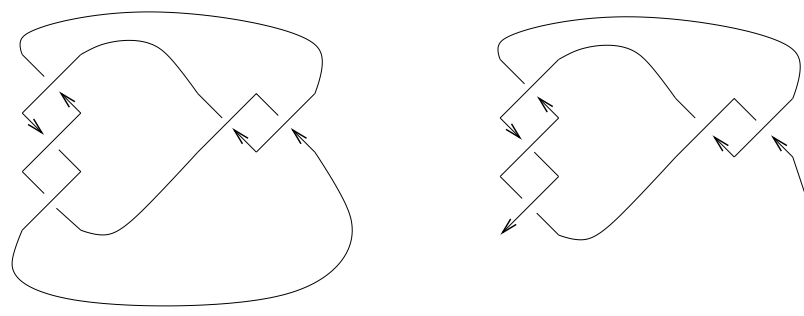

Figure 57. The 2-bridge knot $5_{2}$ and the 2-bridge arc $5_{2}^{\prime}$.

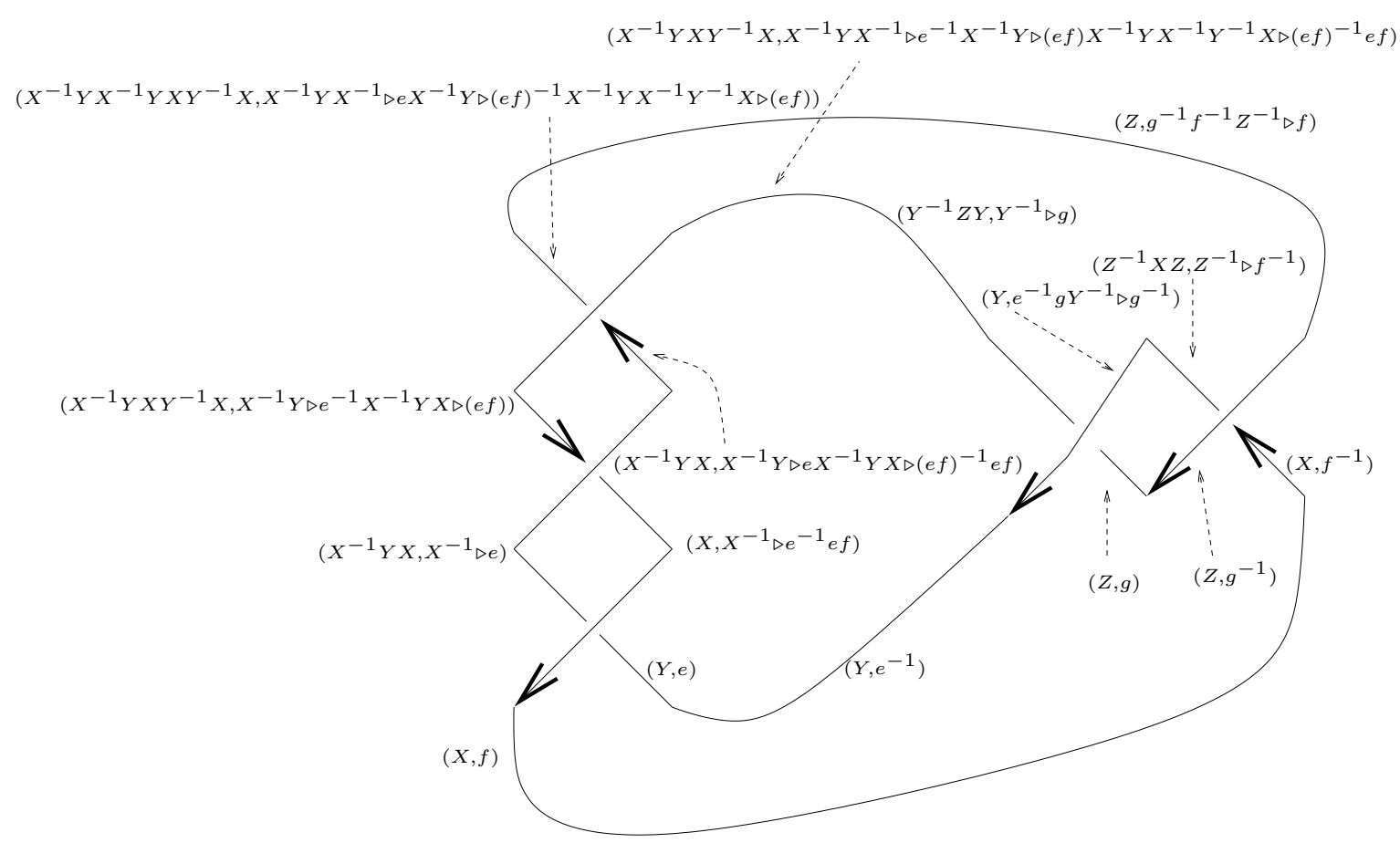

FiguRE 58. Calculation of the crossed module invariant of the 2-bridge knot $5_{2}$.

as it should since the Alexander polynomial of the $5_{2}$ knot is $\Delta\left(5_{2}\right)=2 X^{2}-3+2 X^{-2}$. The formula for the crossed module invariant of the arc $5_{2}^{\prime}$ is

$$
\mathcal{H}_{\mathcal{G}}\left(5_{2}\right)=\# E \#\left\{\begin{array}{l|c}
X, Y \in G ; e, g \in E & \begin{array}{c}
Y X^{-1} Y X Y^{-1} X Y^{-1}=X^{-1} Y X^{-1} Y X Y^{-1} X \\
Y X^{-1} Y X^{-1} \triangleright e^{-1} Y X^{-1} Y \triangleright e Y X^{-1} Y X^{-1} Y^{-1} X \triangleright e^{-1} Y \triangleright e=g^{-1} \\
Z=Y X^{-1} Y X Y^{-1} X Y^{-1} \\
X^{-1} Y X^{-1} \triangleright e X^{-1} Y \triangleright e^{-1} X^{-1} Y X^{-1} Y^{-1} X \triangleright e=g
\end{array}
\end{array}\right\} .
$$

Table 4 compares the values of $\mathcal{H}_{\mathcal{G}_{(n, p)}}\left(5_{2}\right)$ and $\mathcal{H}_{\mathcal{G}_{(n, p)}}\left(c_{1}\left(5_{2}^{\prime}\right)\right)$, for $n=2$ and $p=2,3,4,5,7$. Here, as usual, $c_{1}\left(5_{2}^{\prime}\right)$ is obtained from the welded virtual arc $5_{1}^{\prime}$ by adding a trivial 1-handle to it. In particular it follows that the welded virtual knots $5_{2}=c_{2}\left(5_{2}^{\prime}\right)$ and $c_{1}\left(5_{2}^{\prime}\right)$ are not equivalent, even though they have the same knot groups.

\subsection{The $(n, 2)$-torus knot}

Let $n$ be an odd integer. An analogous calculation as in the case of the trefoil knot and the Solomon seal knot proves that the crossed module invariant of the $(n, 2)$-torus knot $K_{n}$ has the following 


\section{H. Kauffman and J. FARia Martins}

TABLE 4 . The crossed module invariant of the welded virtual knots $5_{2}$ and $c_{1}\left(5_{2}^{\prime}\right)$.

\begin{tabular}{cccccr}
\hline Knot & $\mathcal{H}_{\mathcal{G}_{2,2}}$ & $\mathcal{H}_{\mathcal{G}_{2,3}}$ & $\mathcal{H}_{\mathcal{G}_{2,4}}$ & $\mathcal{H}_{\mathcal{G}_{2,5}}$ & $\mathcal{H}_{\mathcal{G}_{2,7}}$ \\
\hline $5_{2}$ & 24 & 864 & 1536 & 72000 & 987840 \\
$c_{1}\left(5_{2}^{\prime}\right)$ & 24 & 864 & 1536 & 84000 & 1481760 \\
\hline
\end{tabular}

TABLE 5. Comparison between the crossed module invariants of the welded virtual knots $K_{n}$ and $A_{n}$, for $n=3,5, \ldots, 17$.

\begin{tabular}{rrrrrrrrr}
\hline & \multicolumn{1}{c}{$K_{3}$} & \multicolumn{1}{c}{$K_{5}$} & \multicolumn{1}{c}{$K_{7}$} & \multicolumn{1}{c}{$K_{9}$} & \multicolumn{1}{c}{$K_{11}$} & \multicolumn{1}{c}{$K_{13}$} & \multicolumn{1}{c}{$K_{15}$} & \multicolumn{1}{c}{$K_{17}$} \\
\hline $\mathcal{H}_{\mathcal{G}_{(2,3)}}$ & 4320 & 432 & 432 & 4320 & 432 & 432 & 4320 & 432 \\
$\mathcal{H}_{\mathcal{G}_{(2,5)}}$ & 132000 & 168000 & 12000 & 132000 & 12000 & 12000 & 288000 & 12000 \\
\hline & & & & & & & & \\
\hline & $c_{1}\left(A_{3}\right)$ & $c_{1}\left(A_{5}\right)$ & $c_{1}\left(A_{7}\right)$ & $c_{1}\left(A_{9}\right)$ & $c_{1}\left(A_{11}\right)$ & $c_{1}\left(A_{13}\right)$ & $c_{1}\left(A_{15}\right)$ & $c_{1}\left(A_{17}\right)$ \\
\hline $\mathcal{H}_{\mathcal{G}_{(2,3)}}$ & 4752 & 432 & 432 & 4752 & 432 & 432 & 4752 & 432 \\
$\mathcal{H}_{\mathcal{G}_{(2,5)}}$ & 168000 & 204000 & 12000 & 168000 & 12000 & 12000 & 360000 & 12000 \\
\hline
\end{tabular}

expression (in additive notation):

$$
\mathcal{H}_{\mathcal{G}}\left(K_{n}\right)=\#\left\{\begin{array}{c|c}
X, Y \in G ; e, f \in E & \prod_{i=1}^{n} S_{i}=\prod_{i=1}^{n} S_{i+1} \\
\left(\prod_{i=1}^{n} S_{i}\right) \triangleright e-\sum_{k=2}^{n}(-1)^{k}\left(\prod_{i=k}^{n} S_{i}\right) \triangleright(e+f)-f=0
\end{array}\right\} .
$$

Here $S_{i}=X$ if $i$ is even and $S_{i}=Y$ if $i$ is odd. On the other hand, the crossed module invariant of the arc $A_{n}$, obtained from $K_{n}$ in the obvious way (see $\S 3.7$ for the case $n=5$ ) is

$$
\mathcal{H}_{\mathcal{G}}\left(A_{n}\right)=\# E \#\left\{\begin{array}{c|c}
X, Y \in G ; f \in E & \prod_{i=1}^{n} S_{i}=\prod_{i=1}^{n} S_{i+1} \\
-\sum_{k=2}^{n}(-1)^{k}\left(\prod_{i=k}^{n} S_{i}\right) \triangleright f-f=0
\end{array}\right\} .
$$

In Table 5, we compare the value, for each positive odd integer $n \leqslant 17$, of the crossed module invariants $\mathcal{H}_{\mathcal{G}_{(2,3)}}$ and $\mathcal{H}_{\mathcal{G}_{(2,5)}}$ for the pair of welded virtual knots $\left(K_{n}, c_{1}\left(A_{n}\right)\right)$, where $c_{1}\left(A_{n}\right)$ is obtained from $A_{n}$ by adding a trivial 1-handle to it. Since the knot groups of $c_{1}\left(A_{n}\right)$ and of $c_{2}\left(A_{n}\right)=$ $K_{n}$ are isomorphic, this gives some more examples of pairs of one-component welded virtual knots with the same knot group, but distinguished by their crossed module invariant.

\subsection{Final examples}

Let $m$ be a positive integer. We can define an automorphic crossed module $\mathcal{A}_{m}=\left(\mathbb{Z}_{m}, \mathbb{Z}_{2}, \triangleright\right)$, where $\mathbb{Z}_{2}=\{-1,1, \times\}$, and the action of $\mathbb{Z}_{2}$ on $\mathbb{Z}_{m}$ is $1 \triangleright a=a$ and $(-1) \triangleright a=-a$, where $a \in \mathbb{Z}_{m}$. This generalises the crossed module $\mathcal{A}=\mathcal{A}_{3}$ defined in $\S 3.1$.

Consider the link $P$, as well as the associated arc $P^{\prime}$, shown in Figure 59. Let $\mathcal{G}=(E, G, \triangleright)$ be a finite automorphic crossed module with $G$ abelian. An easy calculation shows that

$$
\begin{aligned}
\mathcal{H}_{\mathcal{G}}(P)= & \#\left\{X, Y \in G ; e, f \in E \mid-Y^{-3} X^{-3} \triangleright f+Y^{-3} X^{-2} \triangleright(e+f)-Y^{-2} X^{-2} \triangleright(e+f)\right. \\
& \left.+Y^{-2} X^{-1} \triangleright(e+f)-Y^{-1} X^{-1} \triangleright(e+f)+Y^{-1} \triangleright(e+f)=e\right\} .
\end{aligned}
$$



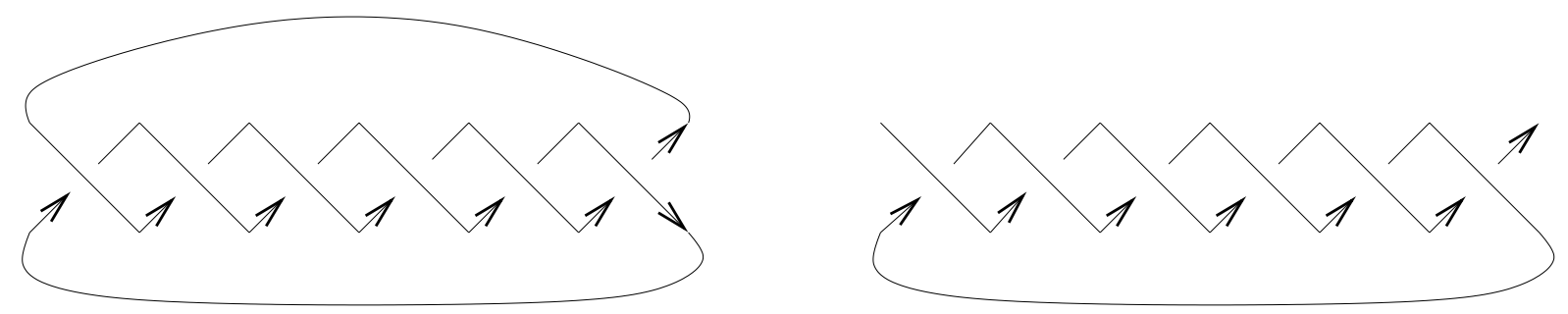

Figure 59. The link $P$ and the associated $\operatorname{arc} P^{\prime}$.

and

$$
\begin{aligned}
\mathcal{H}_{\mathcal{G}}\left(P^{\prime}\right)= & \# E \#\left\{X, Y \in G ; e \in E \mid Y^{-3} X^{-2} \triangleright e-Y^{-2} X^{-2} \triangleright e\right. \\
& \left.+Y^{-2} X^{-1} \triangleright e-Y^{-1} X^{-1} \triangleright e+Y^{-1} \triangleright e=e\right\} .
\end{aligned}
$$

In the case of the automorphic crossed modules $\mathcal{A}_{m}$ defined above, the previous formulae simplify to (for each positive integer $m$ )

$$
\mathcal{H}_{\mathcal{A}_{m}}(P)=m^{2}+2 m \#\left\{a \in \mathbb{Z}_{m} \mid 2 a=0\right\}+m \#\left\{a \in \mathbb{Z}_{m} \mid 6 a=0\right\},
$$

and

$$
\mathcal{H}_{\mathcal{A}_{m}}\left(P^{\prime}\right)=m\left(m+\#\left\{a \in \mathbb{Z}_{m} \mid 2 a=0\right\}+m+\#\left\{a \in \mathbb{Z}_{m} \mid 6 a=0\right\}\right),
$$

thus $\mathcal{H}_{\mathcal{A}}(P)=24$ and $\mathcal{H}_{\mathcal{A}}\left(P^{\prime}\right)=30$. Here as usual $\mathcal{A}=\left(\mathbb{Z}_{3}, \mathbb{Z}_{2}, \triangleright\right)$.

Let $c_{1}\left(P^{\prime}\right)$ be the welded virtual link obtained by adding a trivial 1-handle to the unclosed component of $P^{\prime}$ (see $\S 3.5 .3$ ); thus $P^{\prime}$ and $c_{1}\left(P^{\prime}\right)$ have the same crossed module invariant; see $\S 3.5 .4$. Hence $\left(P=c_{2}\left(P^{\prime}\right), c_{1}\left(P^{\prime}\right)\right)$ is a pair of welded virtual links with the same knot group (see $\S 3.5 .3$ ), but distinguished by their crossed module invariant $\mathcal{H}_{\mathcal{G}}$, where $\mathcal{G}=(E, G, \triangleright)$ is a finite automorphic crossed module, which can be chosen so that $G$ is abelian. In particular we have $\operatorname{Alex}(P) \cong \operatorname{Alex}\left(c_{1}\left(P^{\prime}\right)\right)$, but $\mathrm{CM}(P) \varsubsetneqq \mathrm{CM}\left(c_{1}\left(P^{\prime}\right)\right)$; see $\S 3.3$.

Exercise 6 . Prove directly that $P$ and $c_{1}\left(P^{\prime}\right)$ have the same knot group and are distinguished by their crossed module invariant.

Exercise 7. The previous example can be generalised. For each positive odd integer $n$, let $P_{n}$ be the three-dimensional torus link in $S^{3}$ with $2 n$ crossings, similar to the link $P$ in Figure 59 ; in other words, $P_{n}$ is the $(2,2 n)$-torus link. Let also $P_{n}^{\prime}$ be the associated arc, and let $c_{1}\left(P_{n}^{\prime}\right)$ be the welded virtual link obtained by adding a trivial 1-handle to the unclosed component of $P_{n}^{\prime}$; see Figures 59 and 60 for the case $n=6$. Prove that for any automorphic crossed module $\mathcal{G}=(E, G, \triangleright)$, with $G$ abelian, we have that

$$
\begin{aligned}
\mathcal{H}_{\mathcal{G}}\left(P_{n}\right)=\#\{ & X, Y \in G ; e, f \in E \mid-X^{-n} Y^{-n} \triangleright f \\
& \left.+\sum_{k=1}^{n-1}(X Y)^{-k} \triangleright\left(Y^{-1} \triangleright(e+f)-(e+f)\right)+Y^{-1} \triangleright(e+f)=e\right\}
\end{aligned}
$$

and

$$
\mathcal{H}_{\mathcal{G}}\left(P_{n}^{\prime}\right)=\#\left\{X, Y \in G ; e \in E \mid \sum_{k=1}^{n-1}(X Y)^{-k} \triangleright\left(Y^{-1} \triangleright e-e\right)+Y^{-1} \triangleright e=e\right\} .
$$

Thus if $n$ is odd then we have

$$
\mathcal{H}_{\mathcal{A}_{m}}\left(P_{n}\right)=m^{2}+2 m \#\left\{a \in \mathbb{Z}_{m} \mid 2 a=0\right\}+m \#\left\{a \in \mathbb{Z}_{m} \mid 2 n a=0\right\}
$$



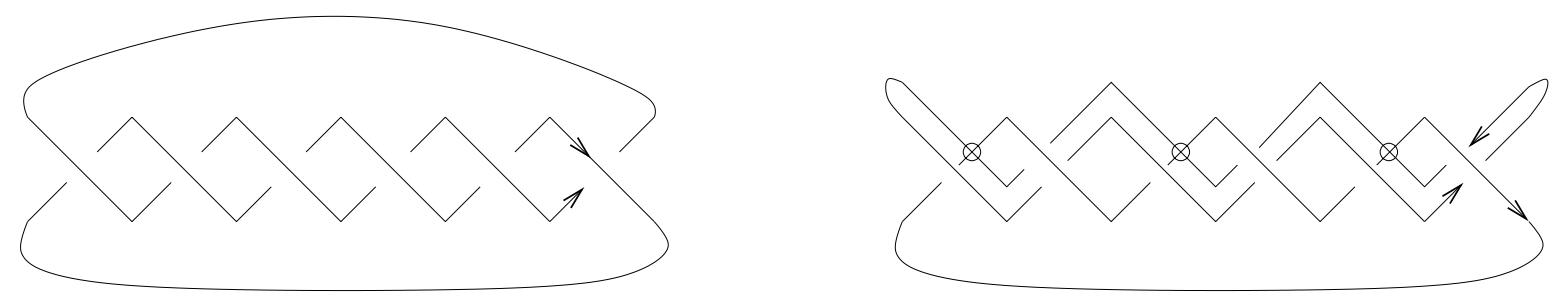

Figure 60 . Two virtual links, $P=c_{2}\left(P^{\prime}\right)$ and $c_{1}\left(P^{\prime}\right)$, with the same knot group but distinguished by their crossed module invariant.

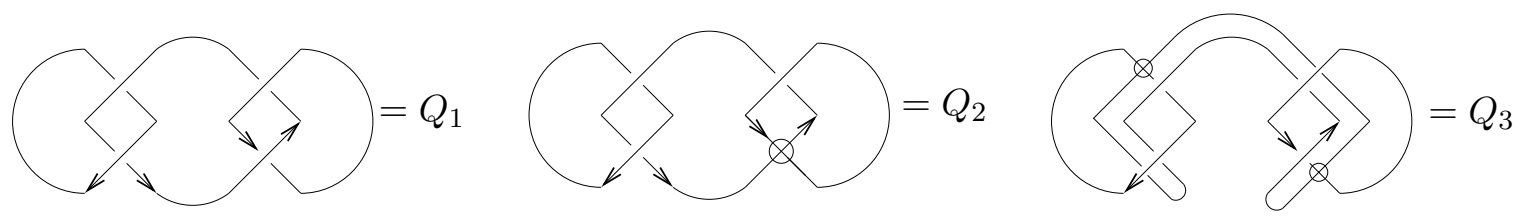

FiguRE 61 . Three virtual links with the same knot group but distinguished by their crossed module invariant.

and

$$
\mathcal{H}_{\mathcal{A}_{m}}\left(P_{n}^{\prime}\right)=m\left(m+\#\left\{a \in \mathbb{Z}_{m} \mid 2 a=0\right\}+m+\#\left\{a \in \mathbb{Z}_{m} \mid 2 n a=0\right\}\right),
$$

where as usual $\mathcal{A}_{m}=\left(\mathbb{Z}_{m}, \mathbb{Z}_{2}, \triangleright\right)$ and $m$ is a positive integer. In particular it follows that

$$
\mathcal{H}_{\mathcal{A}_{n}}\left(P_{n}\right)=2 n^{2}+2 n
$$

and

$$
\mathcal{H}_{\mathcal{A}_{n}}\left(c_{1}\left(P_{n}^{\prime}\right)\right)=\mathcal{H}_{\mathcal{A}_{n}}\left(P_{n}^{\prime}\right)=3 n^{2}+n .
$$

This provides an infinite sequence $\left(P_{n}, c_{1}\left(P_{n}^{\prime}\right)\right)$, where $n$ is an odd integer, of pairs of twocomponent welded virtual links with the same knot group, but distinguished by their crossed module invariant. This sequence includes not only the previous example, but also the case of the Hopf link and the virtual Hopf link in $\S 3.1$.

Note that, taking tubes, the previous example gives an infinite set of pairs of non-isotopic embeddings of a disjoint union of two tori $S^{1} \times S^{1}$ into $S^{4}$ with the same fundamental group of the complement, but distinguished by their crossed module invariant $I_{\mathcal{G}}$ of [FM07a].

Another interesting example is provided by the virtual links $Q_{1}, Q_{2}$ and $Q_{3}$ shown in Figure 61 . The knot groups of $Q_{1}, Q_{2}$ and $Q_{3}$ are all isomorphic to $\{X, Y, Z: X Y=Y X, Z Y=Y Z\}$.

Let $\mathcal{G}=(E, G, \triangleright)$ be an automorphic crossed module. A simple calculation shows that

$$
\begin{aligned}
& \mathcal{H}_{\mathcal{G}}\left(Q_{1}\right)=\#\left\{\begin{array}{l|l}
X, Y, Z \in G ; e, f, g \in E & \begin{array}{l}
-Y^{-1} X^{-1} \triangleright f+Y^{-1} \triangleright(e+f)=e \\
-Z^{-1} Y \triangleright g+Z^{-1} \triangleright(-f+g)=-f
\end{array}
\end{array}\right\}, \\
& \mathcal{H}_{\mathcal{G}}\left(Q_{2}\right)=\#\left\{X, Y, Z \in G ; e, f, g \in E \mid \begin{array}{c}
-Y^{-1} X^{-1} \triangleright f+Y^{-1} \triangleright(e+f)=e \\
Z^{-1} \triangleright f=f
\end{array}\right\}
\end{aligned}
$$

and

$$
\mathcal{H}_{\mathcal{G}}\left(Q_{3}\right)=\#\left\{X, Y, Z \in G ; e, f, g \in E \mid \begin{array}{c}
Y^{-1} \triangleright e=e \\
-Z^{-1} Y \triangleright g+Z^{-1} \triangleright g=0
\end{array}\right\} .
$$

Therefore the crossed module invariant $\mathcal{H}_{\mathcal{A}}$, where as usual $\mathcal{A}=\left(\mathbb{Z}_{3}, \mathbb{Z}_{2}, \triangleright\right)$, separates these $Q_{1}, Q_{2}$ and $Q_{3}$. 


\section{INVARIANTS OF WELDED VIRTUAL KNOTS}

\section{ACKNowledgements}

JFM was financed by Fundação para a Ciência e Tecnologia (Portugal), post-doctoral grant number $\mathrm{SFRH} / \mathrm{BPD} / 17552 / 2004$, part of the research project POCI/MAT/60352/2004 ('Quantum Topology'), also financed by FCT, cofinanced by the European Community fund FEDER. LHK thanks the National Science Foundation for support under NSF Grant DMS-0245588.

\section{REFERENCES}

BM06 J. W. Barrett and M. Mackaay, Categorical representations of categorical groups, Theory Appl. Categ. 16 (2006), 529-557 (electronic).

Bro99 R. Brown, Groupoids and crossed objects in algebraic topology, Homology Homotopy Appl. 1 (1999), 1-78 (electronic).

BZ03 G. Burde and H. Zieschang, Knots, second edition, de Gruyter Studies in Mathematics, vol. 5 (Walter de Gruyter, Berlin, 2003).

CKS04 S. Carter, S. Kamada and M. Saito, Surfaces in 4-space, in Low-dimensional topology, vol. III, Encyclopaedia of Mathematical Sciences, vol. 142 (Springer, Berlin, 2004).

FM07a J. Faria Martins, Categorical groups, knots and knotted surfaces, J. Knot Theory Ramifications 16 (2007), 1181-1217.

FM07b J. Faria Martins, On the homotopy type and the fundamental crossed complex of the skeletal filtration of a CW-complex, Homology Homotopy Appl. 9 (2007), 295-329.

FMP07 J. Faria Martins and T. Porter, On Yetter's invariant and an extension of Dijkgraaf-Witten invariant to categorical groups, Theory Appl. Categ. 18 (2007), 118-150.

FM08 J. Faria Martins, The fundamental crossed module of the complement of a knotted surface, Preprint (2008), arXiv:0801.3921v1 [math.GT].

Fox62 R. H. Fox, A quick trip through knot theory, in Topology of 3-manifolds and related topics, University of Georgia Institute, 1961 (Prentice-Hall, Englewood Cliffs, NJ, 1962), 120-167.

FRR97 R. Fenn, R. Rimányi and C. Rourke, The braid-permutation group, Topology 36 (1997), 123-135.

FY89 P. J. Freyd and D. N. Yetter, Braided compact closed categories with applications to low-dimensional topology, Adv. Math. 77 (1989), 156-182.

Kam07 S. Kamada, Braid presentation of virtual knots and welded knots, Osaka J. Math. 44 (2007), 441-458.

Kau87 L. H. Kauffman, On knots, Annals of Mathematics Studies, vol. 115 (Princeton University Press, Princeton, NJ, 1987).

Kau99 L. H. Kauffman, Virtual knot theory, European J. Combin. 20 (1999), 663-690.

KL06 L. H. Kauffman and S. Lambropoulou, The L-move and virtual braids, J. Knot Theory Ramifications 15 (2006), 773-811.

PS85 S. P. Plotnick and A. I. Suciu, k-invariants of knotted 2-spheres, Comment. Math. Helv. 60 (1985), 54-84.

Por96 T. Porter, Interpretations of Yetter's notion of G-coloring: simplicial fibre bundles and non-abelian cohomology, J. Knot Theory Ramifications 5 (1996), 687-720.

Por98 T. Porter, Topological quantum field theories from homotopy n-types, J. London Math. Soc. (2) 58 (1998), 723-732.

RT90 N. Yu. Reshetikhin and V. G. Turaev, Ribbon graphs and their invariants derived from quantum groups, Comm. Math. Phys. 127 (1990), 1-26.

Ros74 D. Roseman, Woven knots are spun knots, Osaka J. Math. 11 (1974), 307-312.

Sat00 S. Satoh, Virtual knot presentation of ribbon torus-knots, J. Knot Theory Ramifications 9 (2000), $531-542$.

Yaj62 T. Yajima, On the fundamental groups of knotted 2-manifolds in 4-space, J. Math., Osaka City Univ. 13 (1962), 63-71. 


\section{INVARIANTS OF WELDED VIRTUAL KNOTS}

Yet89 D. N. Yetter, Category theoretic representations of knotted graphs in $S^{3}$, Adv. Math. 77 (1989), $137-155$.

Yet93 D. N. Yetter, TQFT's from homotopy 2-types, J. Knot Theory Ramifications 2 (1993), 113-123.

Louis H. Kauffman kauffman@uic.edu

University of Illinois at Chicago, 851 South Morgan St., Chicago, IL 60607-7045, USA

João Faria Martins jmartins@math.ist.utl.pt

Departamento de Matemática, Instituto Superior Técnico (Universidade Técnica de Lisboa), Av. Rovisco Pais, 1049-001 Lisboa, Portugal

Also at Departamento de Matemática, Universidade Lusófona de Humanidades e Tecnologia, Av. do Campo Grande 376, 1749-024, Lisboa, Portugal 